\title{
Effect of small anisotropy and absorption on metamaterial applications: "non-ideal" features of propagation and tunneling of electromagnetic waves
}

\author{
Evgenii Starodubtsev ${ }^{*}$ \\ Gomel State Technical University, October ave. 48, Gomel 246746, Belarus
}

Received: 13 June 2017 / Accepted: 4 December 2017

\begin{abstract}
For cases of isotropic, uniaxial, and biaxial electromagnetic metamaterials (MM), a comparative analysis of the effect of small deviations of local material parameters from "ideal" values on the realization of MM applications ("zero" media, the Veselago-Pendry superlens) has been carried out. On the basis of the detailed investigation of the solutions of dispersion equations, it is established that even a very small dielectric and (or) magnetic anisotropy of a general form is the universal "non-ideal" factor determining (to a much greater extent than small losses) the operability of those MM applications where the wave misphasing in the effective medium is undesirable. The characteristics of wave attenuation in the absorbing isotropic and weakly anisotropic MM are mainly comparable for the applications. Limitations of the traditional approaches using the second-order curves (or surfaces) for analytic modeling of the absorbing MM dispersion equations are shown.
\end{abstract}

\section{Introduction}

The main ideas and experimental realization of the composite electromagnetic materials those macroscopic properties are defined by the elements of their inner microstructure (metamaterials $(\mathrm{MM})$ ) [1-3] have led to the rapid development of MM theory and applications during this century (see, e.g., recent reviews [4-10] and numerous references in these works). Among the promising and actively discussed MM applications, there are development of the "superlens" (the Veselago-Pendry lens, SL) with resolution higher than the diffraction limit, usage of MM with the unique optical properties including negative, near to zero or extremely large electromagnetic material parameters, creation of the various technologies for controlling the visibility of micro- and macroscopic objects, integral optics, information technologies, and many others [4-12].

The majority of the realized electromagnetic MM are characterized by optical absorption or losses, and composite MM are often effectively anisotropic (to a greater or lesser extent) in their dielectric and (or) magnetic properties. The production of isotropic magnetic MM in the optical range is a difficult technological problem [1315]. On the other hand, it is well known that namely a "nonidealness" of the effective electromagnetic characteristics according to various criteria is one of the main factors limiting the performance capabilities of MM applications.

\footnotetext{
* e-mail: starodub@tut. by
}

So, optical absorption worsens considerably the resolution of isotropic SL [3,16-18], leads to wave misphasing and deterioration of the image [19]. As shown in paper [20], even a very small uniaxial optical anisotropy of a general form is a more important problem for SL than absorption. It is caused by the fact that the misphasing of evanescent waves (that are responsible for "superresolution") even in the weakly anisotropic media increases rapidly with increasing the transversal component of wave vector. The opposite situation takes place for absorbing isotropic media: the evanescent wave phase shift decreases with increasing this wave vector component $[19,20]$. Undesirable effects of the phase shift in wave propagation limit also the use of "zero" media (epsilon-mu-near-zero (EMNZ), epsilon-near-zero (ENZ)) that are promising for many integral optics applications [9,21-24].

Moreover, the account of interconnected effects of absorption (losses) and anisotropy on propagation and attenuation of electromagnetic waves is also important for the MM applications. Such investigations were performed in many works only for some specific cases and concrete MM realizations (see, e.g., [25-30]). However, it is interesting to carry out the general comparative analysis of the "non-ideal" (from viewpoint of MM applications) features of electromagnetic wave propagation and tunneling for various absorbing MM: isotropic, uniaxial (at arbitrary orientations of the optical axis), and biaxial (at least, for the biaxial MM with most symmetric electromagnetic properties). Electromagnetic waves in such MM are inhomogeneous, and, in the general case, they are not 
reduced to TM and TE waves in the presence of effective anisotropy $[11,20]$. To reveal the general patterns of the phase and amplitude effects of the radiation interaction with the weakly "non-ideal" MM, it is useful to investigate, at first, the features of solutions of the corresponding dispersion equations.

In the work, the exact solutions of the dispersion equations (that describe the complex wave vector components) are investigated analytically and numerically for a number of "non-ideal" effectively isotropic and anisotropic MM with local electromagnetic properties. The analysis demonstrates new features of proper waves $(\mathrm{PW})$, backward waves, wave propagation and tunneling in MM, and the effect of absorption and anisotropy on the MM applications. The results are obtained using the invariant (independent of the coordinate system choice) calculation methods and the refraction vector formalism [31] taking into account absorption of inhomogeneous PW in the media.

The rest of the paper is organized as follows. In Section 2, the general problem statement and main assumptions used in the work are briefly considered. Features of the exact solutions of the dispersion equations for isotropic and anisotropic (uniaxial, biaxial) absorbing MM are investigated in Sections (3)-(5). By way of examples of the important MM applications (SL, EMNZ, ENZ), the general peculiarities of PW misphasing and damping in such materials are ascertained. First of all, these peculiarities are caused by very small deviations of MM effective electromagnetic parameters from the required "ideal" values. Each of Sections (3)-(5) includes an analytical consideration of the corresponding type of MM and a detailed graphical analysis of the effects of small deviations of material parameters, absorption, and anisotropy on the MM applications. The conclusion (Sect. 6) includes a brief discussion of the obtained results and summarizes the paper. The additional material excluded from the main text of the work is contained in Appendix.

\section{Main assumptions}

Let us consider interaction of plane monochromatic waves with effectively homogeneous media characterized by complex tensors of dielectric permittivity, $\varepsilon$, and magnetic permeability, $\mu$. All or some components of tensors $\varepsilon, \mu$ can have the negative real parts, all the imaginary parts of the components are positive (absorbing linear MM). Phase multiplier $\exp \left[i k_{0}(\boldsymbol{m} \boldsymbol{r})-i \omega t\right]$ is used in expressions for the fields where $\boldsymbol{m}=\left(m_{1}, m_{2}, m_{3}\right)=\boldsymbol{k} / k_{0}$ is a complex refraction vector [31], $k$ is a wave vector (vectors are denoted with boldface symbols), $k_{0}=\omega / c$ is a wave number for vacuum, $i^{2}=-1, \boldsymbol{r}=(x, y, z)$ is a radius-vector. Here and below $(\boldsymbol{u v})$ and $[\boldsymbol{u} \times \boldsymbol{v}]$ denote scalar and vector product of some (both real and complex) vectors $\boldsymbol{u}, \boldsymbol{v}$. The denotations $a^{\prime}=\operatorname{Re}(a), a^{\prime \prime}=\operatorname{Im}(a)$ are used for the real and imaginary parts of scalar or vector quantities. The invariant calculation methods [31] are applied partially in the present work. At first, the invariant relations are considered, and then the results are investigated in the chosen coordinate system where $\boldsymbol{m}=\left(m_{1}, 0, m_{3}\right)$, that is, plane XZ is assumed to be the incidence plane. Meanwhile, the transversal component of refraction vector is supposed to be real $\left(m_{1}\right.$ does not change at different boundaries in solutions of the corresponding boundary problems) and the longitudinal component $m_{3}$ is complex. The usage of the chosen coordinate system is caused by the necessity of obtaining the resulting expressions that include explicitly the components of tensors $\varepsilon, \mu$, anisotropy and absorption parameters for the following analysis of the typical for MM cases of negative or small real parts of the tensors $\varepsilon, \mu$ components.

The validity of material equations $\boldsymbol{D}=\varepsilon \boldsymbol{E}, \boldsymbol{B}=\mu \boldsymbol{H}$ for the effective medium and Maxwell's equations for monochromatic waves [31]

$$
\boldsymbol{E}=-\varepsilon^{-1}[\boldsymbol{m} \times \boldsymbol{H}], \quad \boldsymbol{H}=\mu^{-1}[\boldsymbol{m} \times \boldsymbol{E}],
$$

where $\varepsilon^{-1}, \mu^{-1}$ are inverse to $\varepsilon, \mu$ tensors, is also assumed. Equations (1) and the following below ones are true for both homogeneous and inhomogeneous waves (when the vectors corresponding to the real, $\boldsymbol{m}^{\prime}$, and imaginary, $\boldsymbol{m}^{\prime \prime}$, parts of vector $\boldsymbol{m}$ can be non-parallel). Quantities $\boldsymbol{m}^{\prime}$ and $m^{\prime \prime}$ describe refraction and damping of the electromagnetic wave propagating in an absorbing medium, respectively.

We will use the accurate relations considered below for the graphical analysis of dispersion dependences: equations (2) and (3) for isotropic, equations (13) and (14) for uniaxial, and equation (24) for biaxial MM. Let us investigate functions $m_{3}{ }^{\prime}\left(m_{1}\right), m_{3}{ }^{\prime \prime}\left(m_{1}\right), m_{3}{ }^{\prime}\left(m_{3}{ }^{\prime \prime}\right)$ only for the transmitted waves $\left(m_{3}{ }^{\prime \prime}>0\right)$ at small deviations of effective dielectric and magnetic characteristics from the "ideal" values corresponding to the realization of two important $\mathrm{MM}$ applications: EMNZ (ENZ) and SL. We will consider the effects of both propagation (when $\left|m_{1}\right|$ values do not exceed several units) and tunneling of the electromagnetic waves (up to the values $\left|m_{1}\right| \leq 50$ ) in the investigated MM. Correspondingly, we will use two scales of the parameter $m_{1}$ changes in the figures below. The scales will be designated for convenience as "small" (when the graphs specify features of propagation of the waves) and "large" (when evanescent waves are essentially considered).

\section{Isotropic media}

Using the invariant dispersion equation for isotropic magnetic media, $\boldsymbol{m}^{2}=\varepsilon \mu$, one can obtain the following relations for the real and imaginary parts of the longitudinal component of refraction vector in the chosen coordinate system

$$
m_{3}{ }^{\prime}= \pm \frac{(\varepsilon \mu)^{\prime \prime}}{\sqrt{2\left\{m_{1}^{2}-(\varepsilon \mu)^{\prime}+\sqrt{\left[m_{1}^{2}-(\varepsilon \mu)^{\prime}\right]^{2}+\left((\varepsilon \mu)^{\prime \prime}\right)^{2}}\right\}}}
$$

$$
m_{3}^{\prime \prime}= \pm \sqrt{(1 / 2)\left\{m_{1}^{2}-(\varepsilon \mu)^{\prime}+\sqrt{\left[m_{1}^{2}-(\varepsilon \mu)^{\prime}\right]^{2}+\left((\varepsilon \mu)^{\prime \prime}\right)^{2}}\right\}}
$$


As usual, the value of $m_{1}$ is assumed to be given and the sign of quantity $m_{3}{ }^{\prime \prime}$ is chosen according to the causality condition: $m_{3}{ }^{\prime \prime}>0$ for the wave propagating into MM in the positive direction of axis $Z$ with the amplitude decrease determined by parameter $\exp \left(-m_{3}{ }^{\prime \prime} z\right.$ ) (further this wave is named "transmitted"). The sign of quantity $m_{3}$ ' is determined with account of the previously chosen value of $m_{3}{ }^{\prime \prime}$ and the correspondence of the obtained complex value of $m_{3}$ to the dispersion equation. Obviously, parameter $m_{3}{ }^{\prime} z$ determines the phase shift of the propagating wave. The similar order of the sign choice of the $m_{3}{ }^{\prime \prime}, m_{3}{ }^{\prime}$ values is used for uniaxial (Eqs. (12-14)) and biaxial (Eqs. (22) and (24)) media.

According to equations (2) and (3), even functions $m_{3}{ }^{\prime}\left(m_{1}\right)$ and $m_{3}{ }^{\prime \prime}\left(m_{1}\right)$ are near-hyperbolic and nearlinear, correspondingly, and we have the limiting relations $\left|m_{3}^{\prime}\right| \rightarrow 0,\left|m_{3}^{\prime \prime}\right| \rightarrow \infty$ at $\left|m_{1}\right| \rightarrow \infty$. Functions $m_{3}^{\prime}\left(m_{1}\right)$ (at $\left.(\varepsilon \mu)^{\prime \prime} \neq 0\right)$ and $m_{3}^{\prime \prime}\left(m_{1}\right)$ have the single extremum at $m_{1}=0$ (the presence of either maximum or minimum depends on the sign choice for these functions). One can simply show that the intersection of the graph of function $m_{3}{ }^{\prime}\left(m_{1}\right)$ with the abscissa $m_{3}{ }^{\prime}=0$ is impossible. That means the impossibility of transition of the forward wave $\left(m_{3}{ }^{\prime}>0\right)$ into backward one $\left(m_{3}{ }^{\prime}<0\right)$ and vice versa in the isotropic medium with changing parameter $m_{1}$. We also have the relation $2 m_{3}^{\prime} m_{3}^{\prime \prime}=(\varepsilon \mu)^{\prime \prime}$ from the dispersion equation $\boldsymbol{m}^{2}=\varepsilon \mu$, so dependences $m_{3}{ }^{\prime}\left(m_{3}{ }^{\prime \prime}\right)$, $m_{3}^{\prime \prime}\left(m_{3}{ }^{\prime}\right)$ are hyperbolic (at $\left.(\varepsilon \mu)^{\prime \prime} \neq 0\right)$. A decrease (increase) of the wave attenuation in the isotropic absorbing medium thus leads to an increase (decrease) of the phase shift.

Equations (2) and (3) can be transformed to the form of dispersion equations in coordinates $\left(m_{3}{ }^{\prime}, m_{1}\right),\left(m_{3}{ }^{\prime \prime}\right.$, $\left.m_{1}\right)$ :

$$
\begin{gathered}
4\left(m_{3}^{\prime}\right)^{2}\left[\left(m_{3}^{\prime}\right)^{2}+m_{1}^{2}-(\varepsilon \mu)^{\prime}\right]-\left((\varepsilon \mu)^{\prime \prime}\right)^{2}=0 \\
4\left(m_{3}^{\prime \prime}\right)^{2}\left[\left(m_{3}^{\prime \prime}\right)^{2}-m_{1}^{2}+(\varepsilon \mu)^{\prime}\right]-\left((\varepsilon \mu)^{\prime \prime}\right)^{2}=0 .
\end{gathered}
$$

So, even in the simplest case of the isotropic absorbing medium, equations (4) and (5) are not described by the usually used second-order curves (or the second-order surfaces in the general case). Equations (4) and (5) are the fourth-order equations.

Let us consider some particular cases following from equations (2) and (3). The case $m_{3}{ }^{\prime}=0$ (the purely evanescent wave) is realized for all values of $m_{1}$ only under the condition $(\varepsilon \mu)^{\prime \prime}=\varepsilon^{\prime} \mu^{\prime \prime}+\varepsilon^{\prime \prime} \mu^{\prime}=0$ that can be satisfied for absorbing isotropic media $\left(\varepsilon^{\prime \prime}, \mu^{\prime \prime}>0\right)$ at $\varepsilon^{\prime} \mu^{\prime}<0$.

In this case, we have $m_{3}^{\prime \prime}= \pm \sqrt{m_{1}^{2}+\left(\mu^{\prime \prime} / \varepsilon^{\prime \prime}\right)|\varepsilon|^{2}} \neq 0$.

According to equation (2), realization of the condition $\varepsilon^{\prime} \mu^{\prime}<0$ enables to decrease the phase shift (to 0 at $\left.\varepsilon^{\prime} \mu^{\prime \prime}+\varepsilon^{\prime \prime} \mu^{\prime}=0\right)$ in wave propagation. It is of interest for the applications where such phase shift is undesirable (SL, "zero" MM). As follows from equations (2) and (3), both equality $m_{3}$ " $=0$ and the case of strictly linear dependence $m_{3}^{\prime \prime}\left(m_{1}\right)$ (at $m_{1} \leq 0$ or $m_{1} \geq 0$ ) are not realized in the isotropic absorbing media (for both "conventional" media and MM).
For the analysis of a "non-idealness" of the effective dielectric and magnetic properties of isotropic MM, let us assume that quantities $\varepsilon, \mu$ are of the form:

$$
\varepsilon=a+\Delta \varepsilon, \quad \mu=b+\Delta \mu,
$$

where $a, b$ are characteristic ("ideal") real values of $\varepsilon, \mu$ for some effects (particularly, $a=b=0$ for EMNZ, $a=b=-1$ for SL MM), $\Delta \varepsilon, \Delta \mu$ are small complex additions to $a, b$ $(|\Delta \varepsilon|,|\Delta \mu|<<1)$ characterizing the "non-idealness" of MM properties. Deviations $\Delta \varepsilon, \Delta \mu$ from the ideal conditions can be caused by losses, MM fabrication methods and other factors [13-15].

The numerical analysis of equations (2) and (3) shows that under the conditions, e.g., $|\Delta \varepsilon|,|\Delta \mu|<0.1$ even the linear Taylor expansion of quantities $m_{3}{ }^{\prime}, m_{3}{ }^{\prime \prime}$ in terms of parameters $\Delta \varepsilon, \Delta \mu$ ensures the high accuracy. One can obtain in this case

$$
m_{3}{ }^{\prime} \cong \pm \frac{a \Delta \mu^{\prime \prime}+b \Delta \varepsilon^{\prime \prime}}{2 \sqrt{m_{1}^{2}-a b}}, m_{3}^{\prime \prime} \cong \pm \frac{2\left(m_{1}^{2}-a b\right)-a \Delta \mu^{\prime}-b \Delta \varepsilon^{\prime}}{2 \sqrt{m_{1}^{2}-a b}}
$$

Equations (7) describe essentially evanescent wave tunneling in $\mathrm{MM}\left(m_{1}^{2}>a b\right)$. According to equations (7), quantities $m_{3}{ }^{\prime}, m_{3}{ }^{\prime \prime}$ are determined by parameters $a, b, m_{1}$ and only the imaginary (for $m_{3}{ }^{\prime}$ ) or real (for $m_{3}^{\prime \prime}$ ) parts of deviations $\Delta \varepsilon$, $\Delta \mu$. So, for evanescent waves in the isotropic absorbing medium, the phase shift is determined essentially by the absorption parameters $\left(\Delta \varepsilon^{\prime \prime}, \Delta \mu^{\prime \prime}>0\right)$ and the wave attenuation is determined by deviations $\Delta \varepsilon^{\prime}, \Delta \mu^{\prime}$.

Let us consider the form of equations (7) for some important MM applications. We have in the case of ideal EMNZ MM $(a=b=0)$ :

$$
m_{3}{ }^{\prime} \cong 0, \quad m_{3}^{\prime \prime} \cong \pm m_{1},
$$

that is, the phase shift is absent and the wave attenuation parameter is equal in magnitude to the value of $m_{1}$.

For ideal ENZ MM $(a=0, b=1)$

$$
m_{3}^{\prime} \cong \pm \frac{\Delta \varepsilon^{\prime \prime}}{2 m_{1}}, \quad m_{3}^{\prime \prime} \cong \pm \frac{2 m_{1}^{2}-\Delta \varepsilon^{\prime}}{2 m_{1}}
$$

where $m_{1} \neq 0$, we have the strictly hyperbolic dependence $m_{3}{ }^{\prime}\left(m_{1}\right)$ and the difference of the linear and hyperbolic dependences for function $m_{3}{ }^{\prime \prime}\left(m_{1}\right)$. Meanwhile, parameter $\Delta \mu$ has no effect on quantity $m_{3}$ for EMNZ and ENZ MM in the first approximation.

In the case of ideal SL MM $(a=b=-1)$, we have:

$$
m_{3}{ }^{\prime} \cong \pm \frac{-\Delta \varepsilon^{\prime \prime}-\Delta \mu^{\prime \prime}}{2 \sqrt{m_{1}^{2}-1}}, m_{3}^{\prime \prime} \cong \pm \frac{2\left(m_{1}^{2}-1\right)+\Delta \varepsilon^{\prime}+\Delta \mu^{\prime}}{2 \sqrt{m_{1}^{2}-1}},
$$

where $\left|m_{1}\right|>1$, the dielectric and magnetic absorption effects amplify the wave phase shift simultaneously. It is possible to decrease the wave damping at $\Delta \varepsilon^{\prime}+\Delta \mu^{\prime}<0$ in comparison with the more "ideal" case $\Delta \varepsilon^{\prime}=\Delta \mu^{\prime}=0$. 
For nonmagnetic SL (NMSL) $(a=-1, b=1)$

$$
m_{3}^{\prime} \cong \pm \frac{\Delta \varepsilon^{\prime \prime}-\Delta \mu^{\prime \prime}}{2 \sqrt{m_{1}^{2}+1}}, \quad m_{3}^{\prime \prime} \cong \pm \frac{2\left(m_{1}^{2}+1\right)+\Delta \mu^{\prime}-\Delta \varepsilon^{\prime}}{2 \sqrt{m_{1}^{2}+1}}
$$

the full phase shift compensation at $\Delta \varepsilon^{\prime \prime}=\Delta \mu^{\prime \prime}$ and the wave damping decrease at $\Delta \varepsilon^{\prime}>\Delta \mu^{\prime}$ are possible.

In spite of the approximate character, equations (7)(11) (along with the accurate relations (2) and (3)) can be applied for optimization of the devices using EMNZ (ENZ) and SL (NMSL) MM. That is confirmed by the results of the numerical and graphical analysis executed below.

Subject to parity of functions $m_{3}{ }^{\prime}\left(m_{1}\right)$ and $m_{3}{ }^{\prime \prime}\left(m_{1}\right)$ for the isotropic media (Eqs. (2) and (3)), their graphs are symmetric with respect to the ordinate $m_{1}=0$. So, it is enough to consider the effect of only positive values of parameter $m_{1}$ on quantities $m_{3}{ }^{\prime}, m_{3}{ }^{\prime \prime}$.

Figures 1 and 2 illustrate the effect of small values of quantities $\varepsilon^{\prime}, \mu^{\prime}$ (the top panels) and $\varepsilon^{\prime \prime}, \mu^{\prime \prime}$ (the bottom panels) on dependences $m_{3}^{\prime}\left(m_{1}\right), m_{3}{ }^{\prime \prime}\left(m_{1}\right)$ for the weakly absorbing EMNZ and ENZ MM. At the large scale, dependences $m_{3}{ }^{\prime}\left(m_{1}\right)$ are near-hyperbolic (Fig. $1 \mathrm{a}, \mathrm{c}, \mathrm{e}$ and $\mathrm{g}$ ), and the phase shift decreases quickly with the parameter $m_{1}$ growth for both EMNZ and ENZ MM. At the small scale (Fig. 1b, d, f, h), the dependences are different from hyperbolic and have the pronounced maximum at $m_{1}=0$ (the normal incidence of the wave on MM when the boundary problem is solved). For EMNZ MM, values of the maximum at variation of the parametrs $\varepsilon^{\prime}, \mu^{\prime}$ (Fig. 1b) are greater (approximately by a factor of $10^{2}$ ) than ones at the comparable variation of quantities $\varepsilon^{\prime \prime}, \mu^{\prime \prime}$ (Fig. 1d). For ENZ MM, maxima of function $m_{3}{ }^{\prime}\left(m_{1}\right)$ have the close values with changing parameters $\varepsilon^{\prime}$ and $\varepsilon^{\prime \prime}$ (Fig. 1f and h).

The similar variations of quantities $\varepsilon^{\prime}, \mu^{\prime}$ and $\varepsilon^{\prime \prime}, \mu^{\prime \prime}$ affect dependences $m_{3}{ }^{\prime \prime}\left(m_{1}\right)$ (characterising the wave amplitude attenuation) considerably more weakly (Fig. 2). At the large scale, the graphs in Figure 2a are practically coincident and correpond to all the parameters sets given in Figure 2 (for both EMNZ and ENZ, Fig. 2b-e). Dependences $m_{3}{ }^{\prime \prime}\left(m_{1}\right)$ for EMNZ and ENZ MM are slightly different at small values of $m_{1}$. In particular, the variation of quantities $\varepsilon, \mu$ affects parameter $m_{3}{ }^{\prime \prime}$ for ENZ MM at the same values of $m_{1}$ more strongly (Fig. $2 \mathrm{~b}$ and $\mathrm{c}$ in comparison with Fig. 2d and e). The growth of the values of $\varepsilon^{\prime}, \mu^{\prime}\left(\varepsilon ", \mu^{\prime \prime}\right)$ leads to a decrease (increase) of parameter $m_{3}^{\prime \prime}$, correspondingly, at fixed values of $m_{1}$ (Fig. 2b, d, c and e).

The considered features are characteristic and take place at various values of the parameters, e.g., under the condition $\varepsilon^{\prime} \mu^{\prime}<0$ (Fig. 2b, the top curve). At $\varepsilon^{\prime}=\mu^{\prime}=-0.001, \quad-0.01, \quad-0.1$ the graphs of function $m_{3}{ }^{\prime \prime}\left(m_{1}\right)$ are practically invariable in comparison with ones given in Figure 2a, and graphs $m_{3}{ }^{\prime}\left(m_{1}\right)$ became "mirror-image" with respect to the abscissa axis (that is, the transmitted wave becames backward wave).

The graphical analysis also shows that at the relation $(\varepsilon \mu)^{\prime \prime} \approx 0 \quad$ (the values $\left|\varepsilon^{\prime}\right|=\left|\mu^{\prime}\right|=0.001, \quad 0.01, \quad 0.1$, $\varepsilon^{\prime} \mu^{\prime}<0$ are taken) the condition $m_{3}{ }^{\prime} \approx 0$ is satisfied with the very high accuracy up to distinct values of absorption
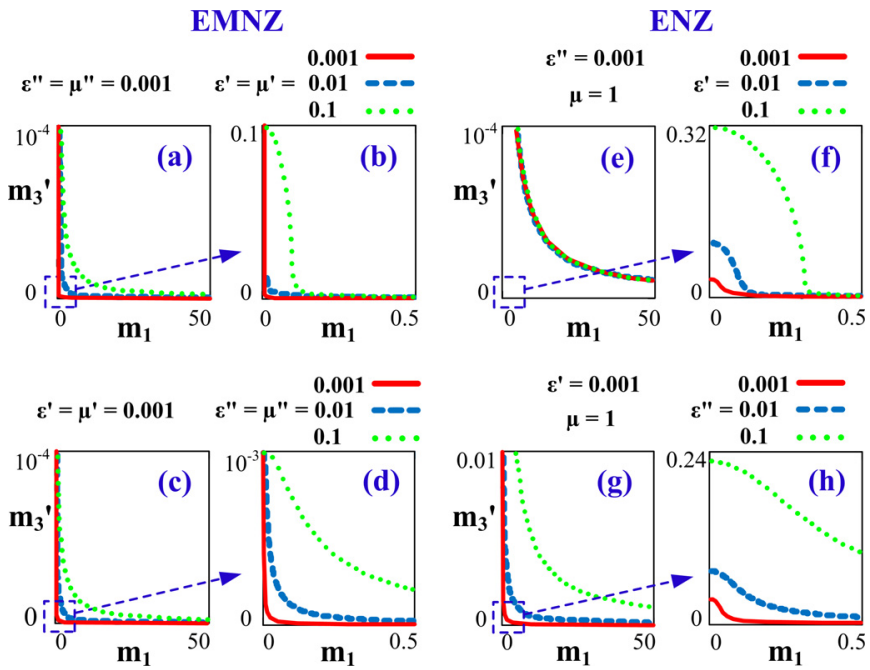

Fig. 1. The effect of small values of quantities $\varepsilon^{\prime}, \mu^{\prime}$ (the top panels) and $\varepsilon^{\prime \prime}, \mu^{\prime \prime}$ (the bottom panels) on dependences $m_{3}{ }^{\prime}\left(m_{1}\right)$ for isotropic EMNZ (a-d) and ENZ (e-h) MM. Figures b, d, f and $\mathrm{h}$ detail the corresponding graphs at small values of $m_{1}$.

parameters of the order of $\varepsilon^{\prime \prime}, \mu^{\prime \prime} \approx 0.1$. In this case, the graphs of function $m_{3}{ }^{\prime \prime}\left(m_{1}\right)$ correspond to ones in Figure 2a.

The results of the graphical analysis which is similar to Figures 1 and 2 data (but for the conditions of isotropic SL and NMSL realization) are given in Figures 3 and 4. According to data in Figure $3 \mathrm{a}-\mathrm{d}$, the transmitted wave in the case of SL is backward $\left(m_{3}{ }^{\prime}<0\right)$, and functions $m_{3}{ }^{\prime}\left(m_{1}\right)$ at varying parameters $\varepsilon^{\prime}, \mu^{\prime}$ and $\varepsilon^{\prime \prime}, \mu^{\prime \prime}$ are characterized by the close values of minima $m_{3}{ }^{\prime}(0)$ in the diapason $(-0.8,-1)$ (Fig. $3 b$ and $d)$. In the NMSL case, the wave is forward $\left(m_{3}^{\prime}>0\right.$, Fig. $\left.3 \mathrm{e}-\mathrm{h}\right)$. At varying parameters $\varepsilon^{\prime}$ and $\varepsilon^{\prime \prime}$, functions $m_{3}{ }^{\prime}\left(m_{1}\right)$ have maxima $m_{3}{ }^{\prime}(0)$ which values differ in approximately two orders (Fig. 3f and h). For both cases (SL, NMSL) the phase shift decreases quickly according to the near-hyperbolic law with the growth of $m_{1}$ up to the large values (Fig. 3a, c, e and $\mathrm{g}$ ). In the case of NMSL, the smaller values of $\left|m_{3}{ }^{\prime}\right|$ in comparison with SL at the same $m_{1}$ values (Fig. 3a, c, and e, g) are realized. In Figure $3 e$ and $f$ the graphs are given for the cases $\varepsilon^{\prime \prime}=0.001, \mu$ " $=0$ ("magnetic" absorption is absent) and $\varepsilon^{\prime \prime}=\mu^{\prime \prime}=0.001$ (the coincident parameters of "dielectric" and "magnetic" absorption that corresponds to the condition $\Delta \varepsilon^{\prime \prime}=\Delta \mu^{\prime \prime}$ in the expression for $m_{3}^{\prime}$ (Eq. (11)). The significant decrease of the phase shift takes place in the last case, as here the condition $(\varepsilon \mu)^{\prime \prime} \approx 0$ is satisfied with the high accuracy.

The graphs in Figure 4 for function $m_{3}{ }^{\prime \prime}\left(m_{1}\right)$ at the SL, NMSL regimes are qualitatively similar to the corresponding graphs in Figure 2 at small values of $m_{1}$ and are practically coincident at the large scale (Fig. 2a and Fig. 4a). However, we have the relations of the parameters describing the given effects at small coincident values of $m_{1}$ : $\left(m_{3}{ }^{\prime \prime}\right)_{S L}<<\left(m_{3}{ }^{\prime \prime}\right)_{E M N Z}$ (Fig. 4b, c and Fig. 2b, c), $\left(m_{3}^{\prime \prime}\right)_{N M S L}>\left(m_{3}^{\prime \prime}\right)_{E N Z}$ (Fig. 4d, e and Fig. 2d, e). At small values of $m_{1}$, varying quantities $\varepsilon^{\prime}, \mu^{\prime}$ affects dependences $m_{3}{ }^{\prime \prime}\left(m_{1}\right)$ for NMSL more strongly in comparison with SL (Fig. 4b and d). Changing $\varepsilon^{\prime \prime}, \mu^{\prime \prime}$ 

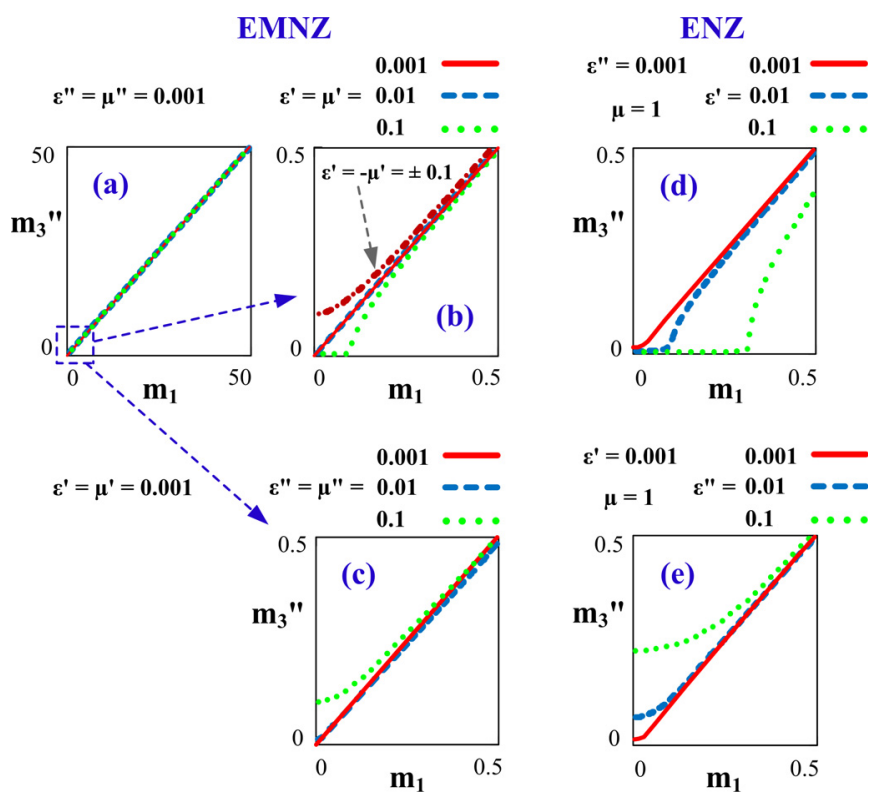

Fig. 2. The effect of small values of quantities $\varepsilon^{\prime}, \mu^{\prime}$ (the top panels) and $\varepsilon^{\prime \prime}, \mu^{\prime \prime}$ (the bottom panels) on dependences $m_{3}{ }^{\prime \prime}\left(m_{1}\right)$ for isotropic EMNZ $(\mathrm{a}-\mathrm{c})$ and $\operatorname{ENZ}(\mathrm{d}, \mathrm{e})$ MM. Figure a corresponds to all the data given in Figures b-e.

values does not affect practically the graphs of function $m_{3}{ }^{\prime \prime}\left(m_{1}\right)$ for NMSL (Fig. 4e) but becomes significantly apparent for SL (Fig. 4c).

The data in Figures 1-4 are represented in Figure 5 in axes $\left(m_{3}{ }^{\prime}, m_{3}{ }^{\prime \prime}\right)$ where the left (Fig. 5a and c) and right (Fig. $5 \mathrm{~b}$ and $\mathrm{d}$ ) panels characterize the effect of varying parameters $\varepsilon^{\prime}, \mu^{\prime}$ and $\varepsilon^{\prime \prime}, \mu^{\prime \prime}$. For the graphs in Figure 5, the removal of the curves from the coordinate origin $\left(m_{3}{ }^{\prime}=\right.$ $\left.m_{3}{ }^{\prime \prime}=0\right)$ corresponds to amplification of the "non-idealness" of the MM effective characteristics. According to data in Figure 5, dependences $m_{3}{ }^{\prime}\left(m_{3}{ }^{\prime \prime}\right)$ are hyperbolic for the isotropic MM, and the absorption parameters effect on $m_{3}{ }^{\prime}\left(m_{3}{ }^{\prime \prime}\right)$ graphs determinatively (especially for ENZ, SL, NMSL MM, Fig. 5b and d). The same changes of parameters $\varepsilon^{\prime}, \mu^{\prime}$ and $\varepsilon^{\prime \prime}, \mu^{\prime \prime}$ lead to the practically coincident $m_{3}{ }^{\prime}\left(m_{3}{ }^{\prime \prime}\right)$ graphs for EMNZ MM and strongly different ones for ENZ MM (Fig. 5a and b). The variation of the values of quantities $\varepsilon^{\prime}, \mu^{\prime}$ effects on dependences $m_{3}{ }^{\prime}\left(m_{3}{ }^{\prime \prime}\right)$ for ENZ, SL, NMSL MM weakly (Fig. 5a and c).

The comparison of the data in Figures 1 -5 shows that the phase shift decrease is accompanied by the increase of the wave damping with the growth of $m_{1}$ (in the general case, $\left.\left|m_{1}\right|\right)$ values. So, for the isotropic absorbing EMNZ (ENZ) and SL (NMSL) MM the phase and amplitude effects actuate mutually antithetically with the growth of transversal wave number $k_{1}=k_{o} m_{1}$ (that is, at the transition to "more evanescent" waves): the phase shift decrease is accompanied by the increase of wave attenuation, and vice versa. The increase of the effective medium absorption leads, as a rule, to the phase shift increase (with the exception of the case $(\varepsilon \mu) " \approx 0$ where the "dielectric" and "magnetic" absorption effects compensate mutually their impact on the phase shift).

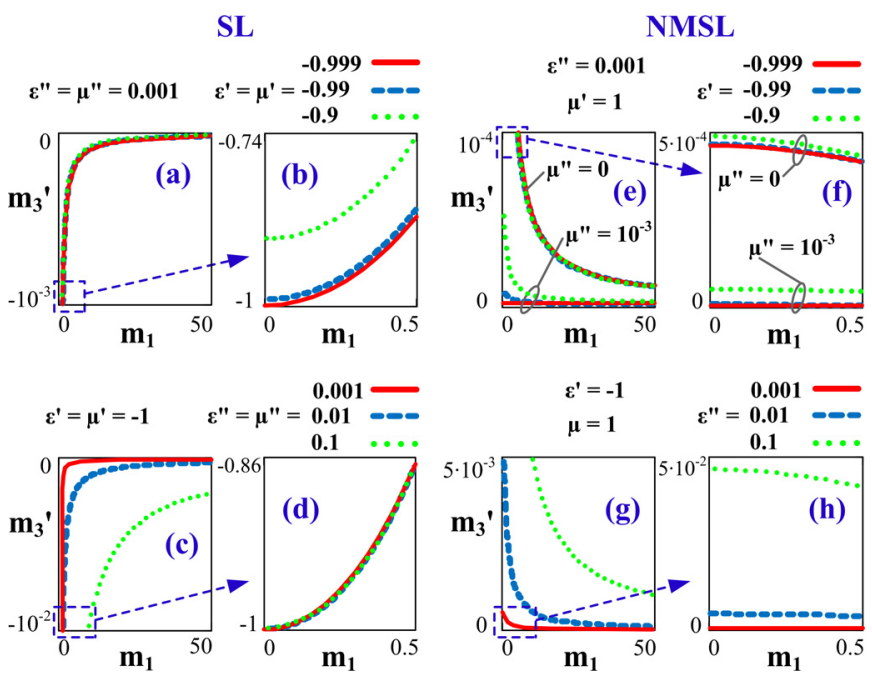

Fig. 3. The effect of small values of quantities $\varepsilon^{\prime}, \mu^{\prime}$ (the top panels) and $\varepsilon^{\prime \prime}, \mu^{\prime \prime}$ (the bottom panels) on dependences $m_{3}{ }^{\prime}\left(m_{1}\right)$ for isotropic SL (a-d) and NMSL (e-h) MM. Figures b, d, f and h detail the corresponding graphs at small values of $m_{1}$.

\section{Uniaxial media}

In the general case of the uniaxial magnetic medium, nondiagonal tensors $\varepsilon, \mu$ have the form [31]: $\varepsilon=\varepsilon_{o}+\left(\varepsilon_{e}-\varepsilon_{o}\right) \boldsymbol{c} . \boldsymbol{c}$, $\mu=\mu_{o}+\left(\mu_{e}-\mu_{o}\right) \boldsymbol{c} . \boldsymbol{c}$ where $\varepsilon_{o}\left(\mu_{o}\right)$ is double singular, $\varepsilon_{e}\left(\mu_{e}\right)$ is non-singular complex eigenvalue of tensor $\varepsilon(\mu)$, $\boldsymbol{c}$ is a unit real vector in the optical axis direction, $\boldsymbol{c} . \boldsymbol{c}$ is a dyadic product. This case is described by the invariant dispersion equations [20]

$$
\begin{aligned}
& \varepsilon_{e}\left(\boldsymbol{m}^{2}-\varepsilon_{o} \mu_{o}\right)=\left(\varepsilon_{e}-\varepsilon_{o}\right)[\boldsymbol{m} \times \boldsymbol{c}]^{2}, \\
& \mu_{e}\left(\boldsymbol{m}^{2}-\varepsilon_{o} \mu_{o}\right)=\left(\mu_{e}-\mu_{o}\right)[\boldsymbol{m} \times \boldsymbol{c}]^{2},
\end{aligned}
$$

corresponding to TM and TE waves only in the particular case of the optical axis orientation in the incidence plane. According to equations (12), the waves in such medium are not strictly propagating or evanescent (for the transmitted waves always $m_{3}{ }^{\prime \prime}>0$ and, in the general case, $m_{3}{ }^{\prime} \neq 0$ even at the incidence on a MM layer of the purely evanescent wave for which $m_{3}{ }^{\prime}=0, m_{3}{ }^{\prime \prime}>0$ ).

Further we use the chosen coordinate system where $\boldsymbol{m}=\left(m_{1}, 0, m_{3}\right), \boldsymbol{c}=(\cos (\alpha), \cos (\beta), \cos (\gamma))$ and consider the first equation from equations (12) corresponding to TM waves in the particular case $\cos (\beta)=0$. The results presented below are analogous to the second equation from equations (12) (corresponding to TE waves at $\cos (\beta)=0)$ at the substitutions $\mu_{o} \leftrightarrow \varepsilon_{o}, \varepsilon_{e} \leftrightarrow \mu_{e}$. Consequently, we obtain the quadratic equation in complex parameter $m_{3}$ with complex coefficients:

$$
\begin{gathered}
{\left[\varepsilon_{o}+\left(\varepsilon_{e}-\varepsilon_{o}\right) \cos ^{2}(\gamma)\right] m_{3}^{2}+2\left(\varepsilon_{e}-\varepsilon_{o}\right) \cos (\alpha) \cos (\gamma) m_{1} m_{3}} \\
+\left[\varepsilon_{o}+\left(\varepsilon_{e}-\varepsilon_{o}\right) \cos ^{2}(\alpha)\right] m_{1}^{2}=\mu_{o} \varepsilon_{o} \varepsilon_{e}
\end{gathered}
$$



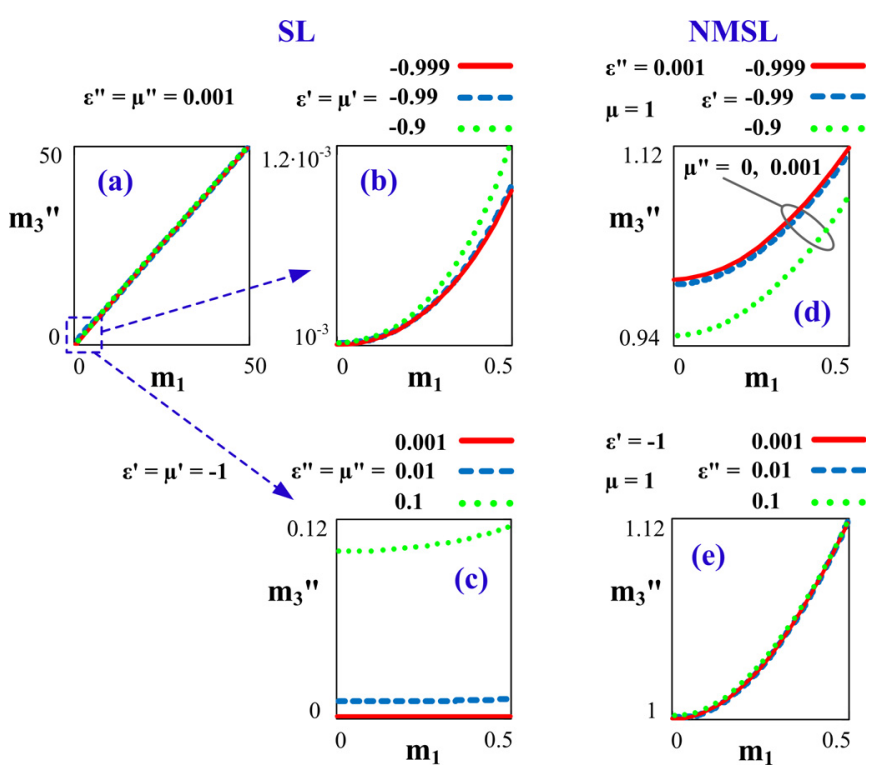

Fig. 4. The effect of small values of quantities $\varepsilon^{\prime}, \mu^{\prime}$ (the top panels) and $\varepsilon^{\prime \prime}, \mu^{\prime \prime}$ (the bottom panels) on dependences $m_{3}{ }^{\prime \prime}\left(m_{1}\right)$ for isotropic SL $(\mathrm{a}-\mathrm{c})$ and NMSL (d, e) MM. Figure (a) corresponds to all the data given in $\mathrm{b}-\mathrm{e}$.

The extraction of the real and imaginary parts in equation (13) leads to a system of two complete fourthorder equations of the form $f\left(m_{3}{ }^{\prime}, m_{1}\right)=0, g\left(m_{3}{ }^{\prime \prime}, m_{1}\right)=0$ both in quantities $m_{3}{ }^{\prime}, m_{3}{ }^{\prime \prime}$ and parameter $m_{1}$. Meanwhile, the solubility conditions for these equations (availability of the real solutions for $m_{3}{ }^{\prime}, m_{3}{ }^{\prime \prime}$ having the physical sense) are identical. The real solutions of these equations can be obtained using the standard analytical method on the basis of the cubic resolvent:

$$
\begin{aligned}
& m_{3}{ }^{\prime}=m_{1} A^{\prime} \pm \sqrt{|B|-B^{\prime}}, \\
& m_{3}{ }^{\prime \prime}=m_{1} A^{\prime \prime} \pm \sqrt{|B|+B^{\prime}},
\end{aligned}
$$

where the denotations are used $\left(p=\varepsilon_{e} / \varepsilon_{o}, q=\varepsilon_{e} \mu_{o}\right)$ :

$$
\begin{gathered}
A=\frac{(1-p) \cos (\alpha) \cos (\gamma)}{1-(1-p) \cos ^{2}(\gamma)}, \\
B=\frac{\left[p+(1-p) \cos ^{2}(\beta)\right] m_{1}^{2}-\left[1-(1-p) \cos ^{2}(\gamma)\right] q}{2\left[1-(1-p) \cos ^{2}(\gamma)\right]^{2}} .
\end{gathered}
$$

According to equations (14), quantities $m_{3}{ }^{\prime}, m_{3}{ }^{\prime \prime}$ are determined by the following parameters: the optical axis direction, quantity $m_{1}$, two complex parameters dependent on the medium material properties, $\varepsilon_{e} / \varepsilon_{o}$ and $\varepsilon_{e} \mu_{o}$. Thus, the waves with the same refraction vector can be excited in the different uniaxial media (characterized by the different parameters $\left.\varepsilon_{o}, \varepsilon_{e}, \mu_{o}\right)$ at the identical aforesaid parameters. It is also followed from equations (14), that functions $m_{3}{ }^{\prime}\left(m_{1}\right)$, $m_{3}{ }^{\prime \prime}\left(m_{1}\right)$ are near-linear, especially with the growth of the $\left|m_{1}\right|$ values corresponding to evanescent waves. So, dependences $m_{3}{ }^{\prime \prime}\left(m_{1}\right)$ for the isotropic and uniaxial media are qualitatively similar, and dependences $m_{3}{ }^{\prime}\left(m_{1}\right)$ are different
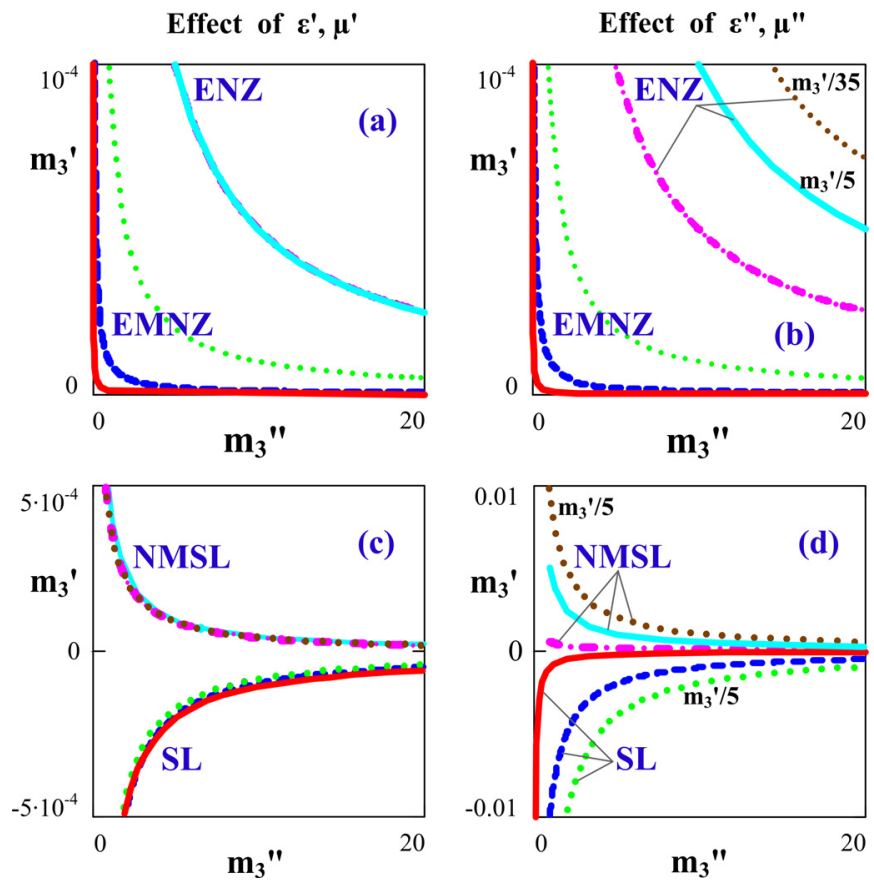

Fig. 5. The effect of small values of quantities $\varepsilon^{\prime}, \mu^{\prime}$ (the left panels) and $\varepsilon^{\prime \prime}, \mu^{\prime \prime}$ (the right panels) on dependences $m_{3}{ }^{\prime}\left(m_{3}{ }^{\prime \prime}\right)$ for the isotropic EMNZ and ENZ (a, b), SL and NMSL (c, d) MM. The corresponding data of Figures 1-4 are taken for the function $m_{3}{ }^{\prime}\left(m_{3}^{\prime \prime}\right)$ graphs.

in essence. With increasing $\left|m_{1}\right|$ values the phase shift of the wave in the isotropic medium decreases quickly according to a near-hyperbolic law, but in the uniaxial medium it increases practically linearly. One can simply show that, in the general case, dependences $m_{3}{ }^{\prime}\left(m_{1}\right), m_{3}{ }^{\prime \prime}\left(m_{1}\right)$ take place in any (non-magnetic, magnetic, MM) uniaxial absorbing medium. Meanwhile, in contrast to the isotropic medium, the accurate (and even approximate, to the first order approximation on the absorption and anisotropy parameters) realization of the condition $m_{3}{ }^{\prime}=0$ at all the parameter $m_{1}$ values is impossible. So, it is impossible to obtain the strictly plane dispersion surface required for many MM applications. According to equations (14), the intersection of the function $m_{3}{ }^{\prime}\left(m_{1}\right)$ graph with the abscissa $m_{3}{ }^{\prime}=0$ is possible. That is one more difference from the isotropic medium case, and it means the possibility of transition of the forward wave into backward one (and vice versa) in the uniaxial medium at changing parameter $m_{1}$ [32].

The important particular case of equations (12) is determined by the condition $[\boldsymbol{m} \times \boldsymbol{c}]^{2}=0$ corresponding to realization of the peculiar PW, the Fedorov - Petrov waves [33] considered for uniaxial MM in [20]. The other interesting cases are realizations of the conditions $\varepsilon_{o}=0$ or $\varepsilon_{e}=0$ in equation (13) corresponding to the cases of "partially absorbing" ENZ MM.

At $\varepsilon_{o}=0$, any values of $\mu_{o}$ and $\varepsilon_{e} \neq 0$, we have from equation (13):

$$
m_{3}=m_{3}^{\prime}=-\frac{\cos (\alpha)}{\cos (\gamma)} m_{1}, \quad m_{3}^{\prime \prime}=0
$$



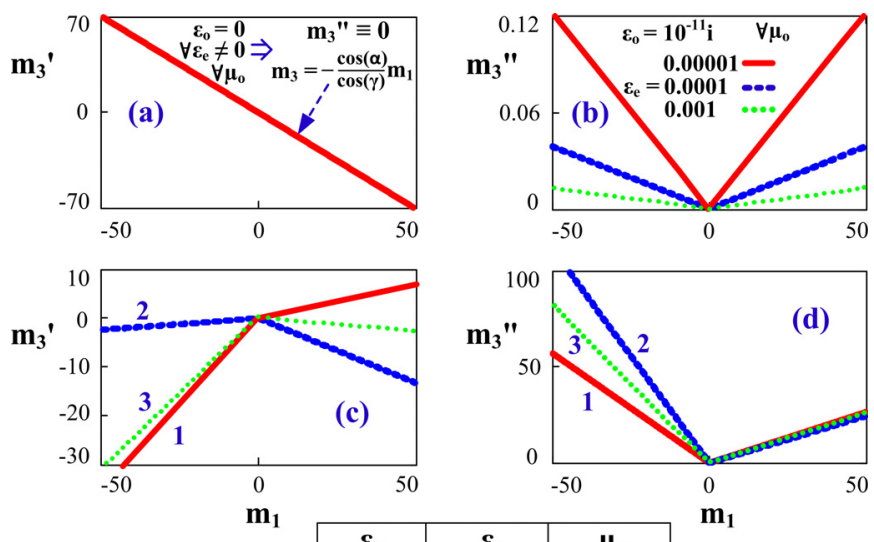

\begin{tabular}{|l|c|c|c|}
\cline { 2 - 4 } \multicolumn{1}{c|}{} & $\boldsymbol{\varepsilon}_{\mathbf{o}}$ & $\boldsymbol{\varepsilon}_{\mathrm{e}}$ & $\boldsymbol{\mu}_{\mathbf{o}}$ \\
\hline 1 & 0.00002 & $0.00001 \mathrm{i}$ & 0 \\
\hline 2 & 0.00001 & $0.00002 \mathrm{i}$ & 0 \\
\hline 3 & 0.00001 & $0.00001 \mathrm{i}$ & 0.00001 \\
\hline
\end{tabular}

Fig. 6. The effect of small birefringence $(a, b)$ and mutual effect of birefringence and dichroism (c, d, values of the parameters for curves 1-3 are given in the table) on dependences $m_{3}{ }^{\prime}\left(m_{1}\right)$ (the left panels) and $m_{3}{ }^{\prime \prime}\left(m_{1}\right)$ (the right panels) for the uniaxial EMNZ MM.

that is, an arbitrarily large MM absorption determined by parameters $\mu_{o}{ }^{\prime \prime}, \varepsilon_{e}{ }^{\prime \prime}$ does not influence quantities $m_{3}{ }^{\prime}, m_{3}{ }^{\prime \prime}$. It is interesting that, according to the relations for $\mathrm{PW}$ polarization in the uniaxial medium [20], vector $\boldsymbol{E}$ is "parallel" to the optical axis in this case (it means that $[\boldsymbol{E} \times \boldsymbol{c}]=0$ with account of complexity of $\boldsymbol{E})$.

At $\varepsilon_{e}=0$, any values of $\mu_{o}$ and $\varepsilon_{o} \neq 0$, one can obtain from equation (13):

$$
m_{3}=\frac{\cos (\alpha) \cos (\gamma) \pm i \cos (\beta)}{1-\cos ^{2}(\gamma)} m_{1}
$$

The feature of equations (15) and (16) is their linearity with respect to quantities $m_{1}, m_{3}$. Meanwhile, they determine the only possible value of $m_{3}^{\prime}$ (and in the case of Eq. (15) also the only value of $m_{3}$, and, therefore, the only PW polarization state) at a given $m_{1}$ for both the transmitted $\left(m_{3}{ }^{\prime \prime}>0\right)$ and reflected $\left(m_{3}{ }^{\prime \prime}<0\right)$ waves characterized by equation (13). One of these waves is forward $\left(m_{3}{ }^{\prime}>0\right)$ and the other is backward $\left(m_{3}{ }^{\prime}<0\right)$ subject to the optical axis orientation. Note, that the similar case of the linear dispersion equation is impossible for the isotropic medium. It is also seen that explicit dependences of quantities $m_{3}{ }^{\prime}$, $m_{3}{ }^{\prime \prime}$ on MM material parameters are vanished in these particular cases, and quantity $m_{3}$ is determined only by the optical axis direction and $m_{1}$ value. Under the additional conditions to the optical axis orientations $\cos (\alpha)=0$, $\cos (\gamma) \neq 0$ (the case of Eq. (15)) or $\cos (\gamma)=\cos (\beta)=0$ (the case of Eq. (16)) such MM are "ideal" ENZ MM: $m_{3}{ }^{\prime}=m_{3}{ }^{\prime \prime}=0$ at any values of $m_{1}$. Thus, for the considered cases (Eqs. (15) and (16)) the dispersion equations are determined only by the optical axis direction that, obviously, depends on tensor $\varepsilon, \mu$ components.
Let us use equation (13) for obtaining the approximate relations that are similar to equations (7) and characterize dependences $m_{3}{ }^{\prime}\left(m_{1}\right), m_{3}{ }^{\prime \prime}\left(m_{1}\right)$ in the first order approximation on the "non-ideal" deviations of the absorption and anisotropy parameters. As in equations (6), we assume that

$$
\varepsilon_{o}=a+\Delta \varepsilon_{o}, \quad \mu_{o}=b+\Delta \mu_{o}, \quad \varepsilon_{e}-\varepsilon_{o}=\Delta \varepsilon,
$$

where $a, b$, are "ideal" (for some effects) real values of quantities $\varepsilon_{o}, \mu_{o}$, small complex parameters $\Delta \varepsilon_{o}, \Delta \mu_{o}, \Delta \varepsilon$ $\left(\left|\Delta \varepsilon_{o}\right|,\left|\Delta \mu_{o}\right|,|\Delta \varepsilon|<<1\right)$ describe small deviations of the MM effective parameters from "ideal" conditions, and $\Delta \varepsilon$ characterizes small dielectric anisotropy. According to the numerical analysis, the condition $\left|\Delta \varepsilon_{o}\right|,\left|\Delta \mu_{o}\right|,|\Delta \varepsilon| \leq 0.1$ ensures the high accuracy again. Consequently, we obtain

$$
\begin{aligned}
& \left(m_{3}{ }^{\prime}\right)_{ \pm} \cong \\
& -\frac{m_{1} \Delta \varepsilon^{\prime} \cos (\alpha) \cos (\gamma)}{a} \pm \frac{\left[b \Delta \varepsilon_{o}+a \Delta \mu_{o}+(Q / a) \Delta \varepsilon\right]^{\prime \prime}}{2 \sqrt{m_{1}^{2}-a b}} \\
& \left(m_{3}{ }^{\prime \prime}\right)_{ \pm} \cong \pm \sqrt{m_{1}^{2}-a b} \\
& -\frac{m_{1} \Delta \varepsilon^{\prime \prime} \cos (\alpha) \cos (\gamma)}{a} \mp \frac{\left[b \Delta \varepsilon_{o}+a \Delta \mu_{o}+(Q / a) \Delta \varepsilon\right]^{\prime}}{2 \sqrt{m_{1}^{2}-a b}}
\end{aligned}
$$

where $m_{1}^{2}>a b, \quad Q=a b \sin ^{2}(\gamma)-m_{1}^{2}\left[\cos ^{2}(\alpha)-\cos ^{2}(\gamma)\right]$, and only the upper or only lower sign is chosen in each relation. Expressions (18) and (19) generalize the relations for SL MM $(a=b=-1)$ [20] in the case of arbitrary real values of $a \neq 0, b$.

At $a=0$ (EMNZ or ENZ MM) the following expression is a highly accurate approximation for parameter $m_{3}$

$$
\text { See equation (20) below: }
$$

According to equation (20), parameter $m_{3}$ is directly proportional to $m_{1}$ and independent of the effective medium magnetic properties.

In the general case of anisotropic media, functions $m_{3}{ }^{\prime}\left(m_{1}\right)$ and $m_{3}{ }^{\prime \prime}\left(m_{1}\right)$ are not appeared to be even (Eqs. (14) and (24)). So, let us consider the effect of not only positive but also negative values of parameter $m_{1}$ in the diapason $\left|m_{1}\right| \leq 50$ on quantities $m_{3}{ }^{\prime}, m_{3}{ }^{\prime \prime}$ for the transmitted waves. For all the graphs in Figures 6-8 the values of the angles $\alpha=\pi / 4, \beta=\gamma=\pi / 3$ determining the optical axis direction in the chosen coordinate system are used. The features considered below take place also for the different orientations of the optical axis.

The graph of linear function $m_{3}^{\prime}\left(m_{1}\right)$ for the case $\varepsilon_{o}=0$ (Eq. (15)) is given in Figure 6a. In this case, the wave amplitude attenuation is absent: $m_{3}{ }^{\prime \prime}=0$. The very small change of parameter $\varepsilon_{o}$ (from 0 to $10^{-11}$ i, Fig. 6b) leads to the appearance of the strong and near-linear dependences $m_{3}{ }^{\prime \prime}\left(m_{1}\right)$ that are very "sensitive" to very small changes of quantity $\varepsilon_{e}$. The

$$
\left(m_{3}\right)_{ \pm} \cong m_{1} \frac{-\Delta \varepsilon \cos (\alpha) \cos (\gamma) \pm \sqrt{-\Delta \varepsilon_{o}\left[\Delta \varepsilon_{o}+\Delta \varepsilon\left(\cos ^{2}(\alpha)+\cos ^{2}(\gamma)\right)\right]}}{\Delta \varepsilon_{o}+\Delta \varepsilon \cos ^{2}(\gamma)} .
$$



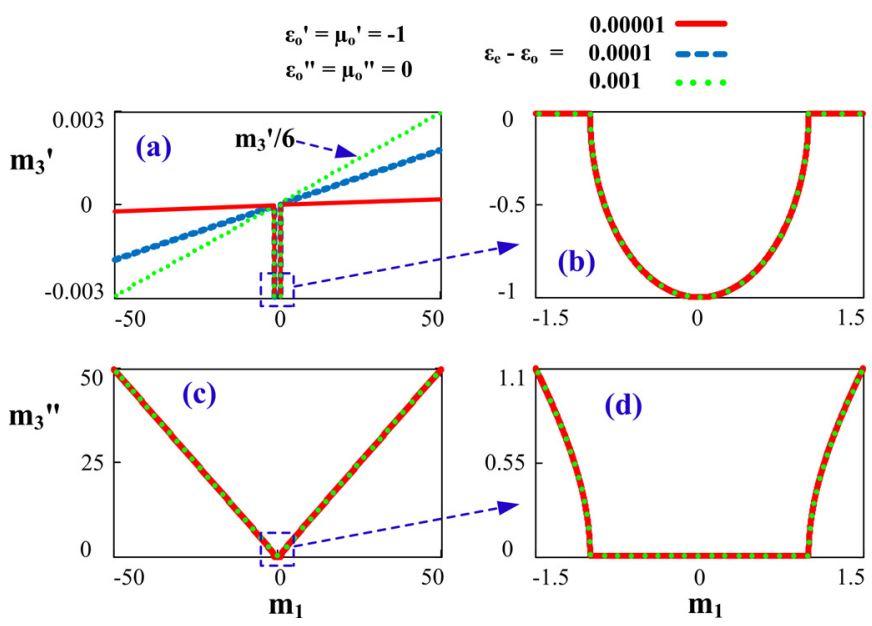

Fig. 7. The effect of small birefringence on dependences $m_{3}{ }^{\prime}\left(m_{1}\right)$ $(\mathrm{a}, \mathrm{b})$ and $m_{3}{ }^{\prime \prime}\left(m_{1}\right)(\mathrm{c}, \mathrm{d})$ for the nonabsorbing uniaxial SL MM. Figures b, d detail the corresponding graphs at small values of $m_{1}$.

simultaneous presence of small birefringence and dichroism (of the order of $\left|\varepsilon_{o}\right|,\left|\varepsilon_{e}\right| \approx 10^{-5}$ ) leads to near to piecewise-linear graphs of functions $m_{3}{ }^{\prime}\left(m_{1}\right)$ and $m_{3}{ }^{\prime \prime}\left(m_{1}\right)$ that are asymmetric with respect to the positive and negative values of $m_{1}$ (Fig. 6c and d). With that, it is possible to change the transmitted wave type (forward, $m_{3}{ }^{\prime}>0$, backward, $m_{3}{ }^{\prime}<0$ ) with varying parameter $m_{1}$ (Fig. 6a and Fig. 6c, curve 1). Also the different character of the changes of dependences $m_{3}{ }^{\prime \prime}\left(m_{1}\right)$ at $m_{1}<0$ and $m_{1}>0$ (Fig. 6d) is possible. The graphs in Figure 6 do not practically change also at the small scale of values of $m_{1}$ and under the condition $\mu_{o}=1$, that is, for the case of weakly anisotropic uniaxial ENZ MM.

According to Figure 6 data, the very small changes of parameters $\varepsilon_{o}, \varepsilon_{e}$ (of the order of $\left|\varepsilon_{o}\right|,\left|\varepsilon_{e}\right| \approx 10^{-5}-10^{-4}$ and less) lead to the significant changes of quantities $m_{3}{ }^{\prime}$ (Fig. 6c) and $m_{3}{ }^{\prime \prime}$ (Fig. 6b and $\mathrm{d}$ and the transition from Fig. 6a to b) for evanescent waves (at $\left|m_{1}\right|>>0$ ). Such features of the considered near to "zero" MM can be of interest for controlling these waves characteristics and sensor applications.

The data in Figures 7 and 8 characterize the effect of parameters of small birefringence (Fig. 7), dichroism (Fig. 8 a and d), birefringence and dichroism (Fig. 8b-d) on the conditions of the SL MM realization (the analogous features take place for NMSL MM). At the large scale of $m_{1}$ values, dependences $m_{3}{ }^{\prime}\left(m_{1}\right), m_{3}{ }^{\prime \prime}\left(m_{1}\right)$ are near-linear (Fig. 7a, c, and Fig. 8a-c), and the graphs $m_{3}{ }^{\prime}\left(m_{1}\right)$ are qualitatively similar to the graphs for EMNZ MM (Fig. 6c). However, the phase shifts are appeared to be in 3-4 order smaller in comparison with the EMNZ MM regime with changing values $m_{1}$ in the case of SL (Fig. 7a and Fig. 8a-c in comparison with Fig. 6a and c). At small $m_{1}$ values, dependences $m_{3}{ }^{\prime}\left(m_{1}\right)$ are nonlinear and symmetric with respect to axis $m_{1}=0$ (Fig. 7b, d, and Fig. 8d). At the large scale, the function $m_{3}{ }^{\prime \prime}\left(m_{1}\right)$ graphs in Figure $7 \mathrm{c}$ coincide and correspond to all the parameters sets given in Figures 7 and 8 . The comparison of the data in Figure $8 \mathrm{a}-\mathrm{c}$ shows that asymmetry of the function $m_{3}{ }^{\prime}\left(m_{1}\right)$ graphs with respect to the positive and negative $m_{1}$ values is determined mainly by the effective medium birefringence. With that, the phase shifts due to birefringence are appeared to be in several times

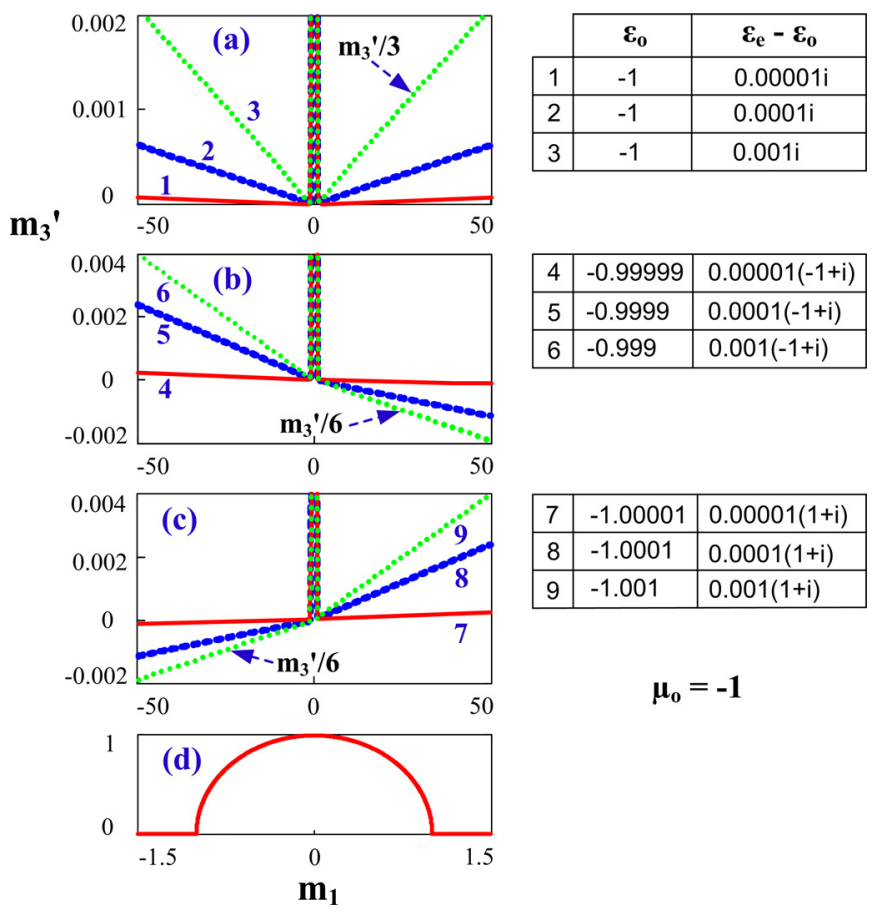

Fig. 8. The effect of small dichroism (a), mutual effect of birefringence and dichroism $(b, c)$ (values of the parameters for curves 1-9 are given in the corresponding tables) on dependences $m_{3}{ }^{\prime}\left(m_{1}\right)$ for the uniaxial SL MM. Figure d corresponds to the cases $(\mathrm{a}-\mathrm{c})$ at small values of $m_{1}$.

greater than ones due to dichroism (at the same values of birefringence, $\left|\varepsilon_{e}{ }^{\prime}-\varepsilon_{o}{ }^{\prime}\right|$, and dichroism, $\left|\varepsilon_{e}{ }^{\prime \prime}-\varepsilon_{o}{ }^{\prime \prime}\right|$, parameters, curves $4-9$ and $1-3$ in Fig. 8 ).

Figure 9 generalizes Figures 6-8 data in the form of dependences $m_{3}^{\prime}\left(m_{3}{ }^{\prime \prime}\right)$. According to Figure 9 graphs at the large scale of $m_{1}$ values (at large $m_{3}{ }^{\prime \prime}$ values), dependences $m_{3}{ }^{\prime}\left(m_{3}{ }^{\prime \prime}\right)$ are near-linear for the weakly uniaxial MM. At small $m_{1}$ values (at small $m_{3}{ }^{\prime \prime}$ values), graphs $m_{3}{ }^{\prime}\left(m_{3}{ }^{\prime \prime}\right)$ are near-linear only for EMNZ MM (for the data in Fig. 9a and b). In the case of SL MM with small uniaxial anisotropy, dependences $m_{3}{ }^{\prime}\left(m_{3}{ }^{\prime \prime}\right)$ can be close to piecewise-linear (Fig. 9d and f) and nonlinear (Fig. 9h). Also the data in Figure 9 illustrate clearly the different scale of parameter $m_{3}{ }^{\prime}$ changes with the growth of the absorption parameters for the considered EMNZ and SL MM.

\section{Biaxial media}

The cases of biaxial absorbing media can be realized in modeling and experimental determination of effective electromagnetic MM characteristics. With that, MM dielectric and magnetic characteristics can demonstrate the different symmetry properties. Consequently, e.g., effective tensors $\varepsilon, \mu$ cannot be reduced to the diagonal form in the same coordinate system. Obviously, the properties of such MM become more complex in comparison with the cases of isotropic and uniaxial media. For such problems (as for cases of "conventional" anisotropic media), the invariant methods of phenomenological electrodynamics $[31,34]$ are high-performance ones. 


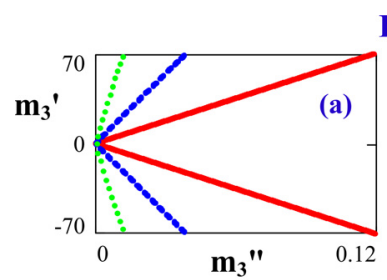

EMNZ

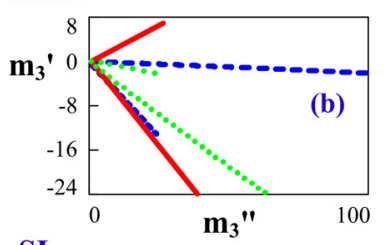

SL
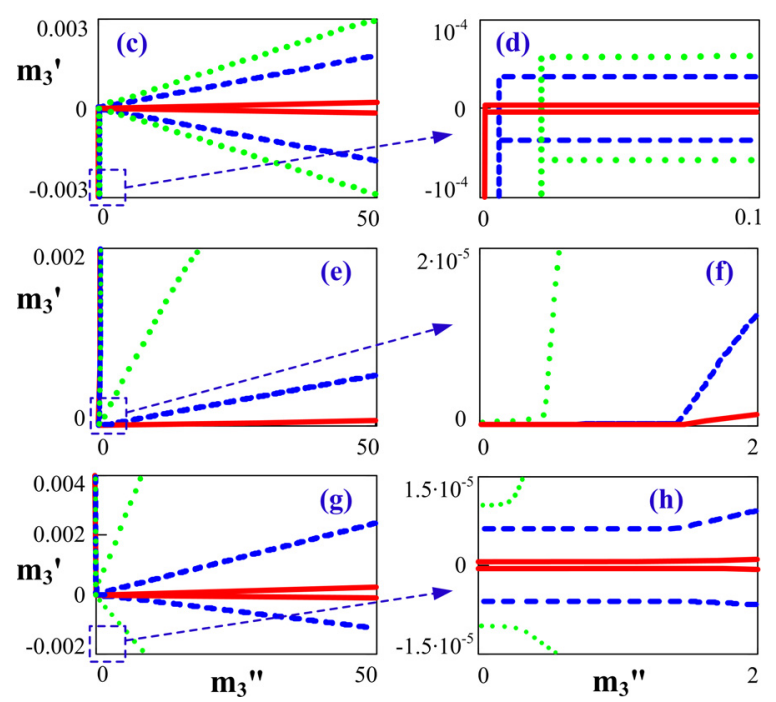

Fig. 9. Dependences $m_{3}{ }^{\prime}\left(m_{3}{ }^{\prime \prime}\right)$ for the data given in Figures 6-8. The data correspondence is: a - Figures $6 \mathrm{a}, \mathrm{b} ; \mathrm{b}-$ Figures $6 \mathrm{c}, \mathrm{d}$; c, $\mathrm{d}-$ Figure $7 \mathrm{a}-\mathrm{d}$; e, f - Figure 8a; g, h - Figure 8b (the same line types are used for the corresponding data). Figures $d, f$ and $h$ detail the data in Figures c, e and $g$ at small values of $m_{3}{ }^{\prime \prime}$.

Meanwhile, as a rule, MM are artificial materials from "meta-atoms" ordered spatially according to some rules, that is, the given materials are more frequently characterized by pronounced symmetry properties. Therefore, let us consider as a biaxial MM model a rather general case of one from the most symmetric configurations such as absorbing magnetic medium of orthorhombic symmetry with the identical structure of effective tensors $\varepsilon, \mu$.

In this case, non-diagonal tensors $\varepsilon, \mu$ are reduced to the diagonal form in the same real coordinate system and can be expressed in the following form [31]:

$$
\varepsilon=\sum_{q=1}^{3} \varepsilon_{q} \boldsymbol{c}_{q} \cdot \boldsymbol{c}_{q}, \quad \mu=\sum_{q=1}^{3} \mu_{q} \boldsymbol{c}_{q} \cdot \boldsymbol{c}_{q},
$$

where $\varepsilon_{q}, \mu_{q}$ are complex scalars (eigenvalues of tensors $\varepsilon$, $\mu), \boldsymbol{c}_{q}$ are unit real vectors $\left(q=1,2,3, c_{q}^{2}=1\right)$ directed along the second order symmetry axes which are mutually orthogonal in the considered media $\left(\boldsymbol{c}_{q}\right.$ are also perpendicular to the planes of the medium mirror symmetry), $\boldsymbol{c}_{q} \cdot \boldsymbol{c}_{q}$ are dyads. For example, for layered MM, one of the vectors $\boldsymbol{c}_{q}$ can be perpendicular to the layers boundaries and two others can lie in the layers plane.

Substitution of expressions (21) into the well-known invariant equation of normals for magnetoanisotropic media characterized by arbitrary tensors $\varepsilon, \mu[31,34]$ leads to the dispersion equation:

$$
\begin{aligned}
& \left(\sum_{q=1}^{3} \varepsilon_{q} M_{q}^{2}\right)\left(\sum_{q=1}^{3} \mu_{q} M_{q}^{2}\right) \\
& \quad-\varepsilon_{1} \varepsilon_{2} \varepsilon_{3}\left[\mu_{1} F_{23} M_{1}^{2}+\mu_{2} F_{13} M_{2}^{2}+\mu_{3} F_{12} M_{3}^{2}-\mu_{1} \mu_{2} \mu_{3}\right] \\
& \quad=0,
\end{aligned}
$$

where the denotations are used: $M_{q}=\left(\boldsymbol{m} \boldsymbol{c}_{q}\right)$, $F_{p r}=\mu_{p} / \varepsilon_{p}+\mu_{r} / \varepsilon_{r}(q, p, r=1,2,3)$.

One can show that only in the particular cases $M_{q}=0$ the left part of equation (22) is representable in the form of the product of two multipliers that are quadratic with respect to refraction vector $\boldsymbol{m}$. Then complex vector $\boldsymbol{m}$ "lies" in one from three principal planes of the medium (one from three scalar products $\left(\boldsymbol{m} \boldsymbol{c}_{q}\right)$ is equal to 0$)$. Only in these cases it is possible to separate $\mathrm{PW}$ in the medium into two groups corresponding to TM and TE waves at the transition to diagonal tensors $\varepsilon, \mu$.

One more particular case of equation (22) is realized under the condition $\varepsilon=K \mu$ where $K$ is a complex scalar (direct proportionality of tensors $\varepsilon$ and $\mu$, the onerefringent medium case [31]). Under this condition, equation (22) leads to the relations

$$
\left(\sum_{q=1}^{3} \varepsilon_{q} M_{q}^{2}\right)-\mu_{1} \varepsilon_{2} \varepsilon_{3}=\left(\sum_{q=1}^{3} \mu_{q} M_{q}^{2}\right)-\varepsilon_{1} \mu_{2} \mu_{3}=0 .
$$

In this case, refraction vector depends on the direction of the wave propagation but it does not depend on the wave polarization (birefringence is absent) [31].

At the transition to the chosen coordinate system where $\boldsymbol{m}=\left(m_{1}, 0, m_{3}\right), \boldsymbol{c}_{q}=\left(\cos \left(\alpha_{q}\right), \cos \left(\beta_{q}\right), \cos \left(\gamma_{q}\right)\right)$ equation (22) leads to the complete fourth-order equation in quantity $m_{3}$ (and also in $m_{1}$ )

$$
A m_{3}^{4}+B m_{3}^{3}+C m_{3}^{2}+D m_{3}+E=0,
$$

where the expressions for coefficients $A, B, C, D, E$ are given in Appendix.

Extraction of the real and imaginary parts of equation (24) leads to the system of two sufficiently awkward equations and each of them is the complete fourth-order equation in quantities $m_{3}{ }^{\prime}, m_{3}{ }^{\prime \prime}, m_{1}$. In the general case, the analytical solution of the given system is seemed to be impossible. Obviously, knowing the coefficients of equation (24), one can obtain complex analytical solutions for quantity $m_{3}$ using the well-known formulas or applying the numerical methods to find four complex roots of the equation. Both these methods (analytical and numerical) of the solution of dispersion equation (24) are used for finding and verification of the values of $m_{3}$. Then the values of $m_{3}{ }^{\prime}, m_{3}{ }^{\prime \prime}$ are chosen with account of execution of the causality conditions and correpondence of the obtained values pairs $\left(m_{3}{ }^{\prime}, m_{3}{ }^{\prime \prime}\right)$ to equation (24).

The results of the graphical analysis of equation (24) under the conditions that are close to realization of EMNZ (Figs. 10 and 13a-c) and SL (Figs. 11, 12, 13d and e) MM are given in Figures 10-13. For visualization, the different 


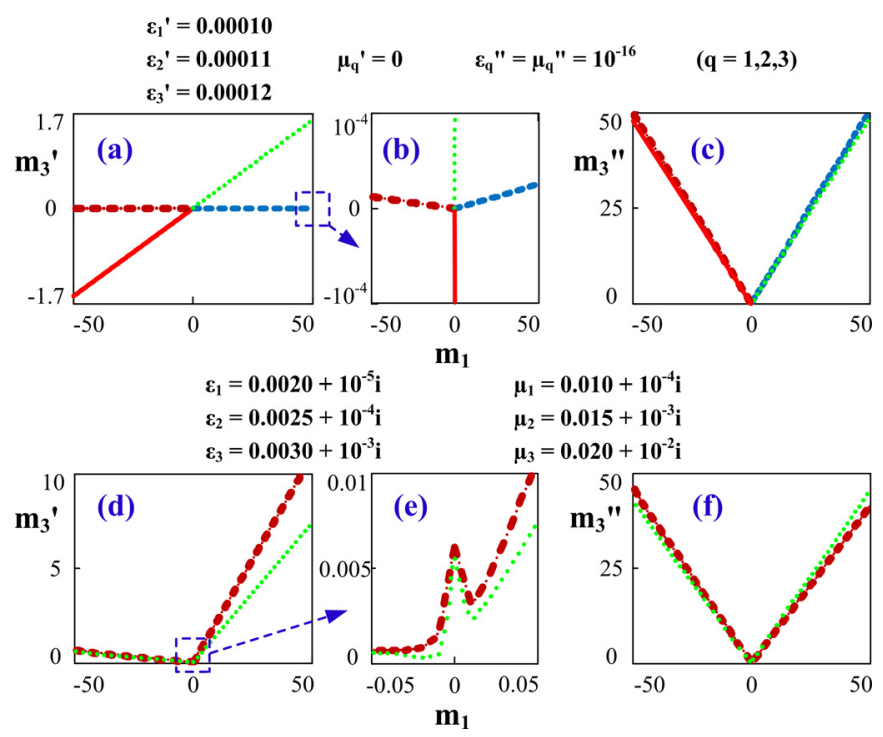

Fig. 10. The effect of small birefringence $(\mathrm{a}-\mathrm{c})$, the mutual effect of birefringence and dichroism (d-f) on dependences $m_{3}{ }^{\prime}\left(m_{1}\right)$ (a, $\mathrm{b}, \mathrm{d}, \mathrm{e})$ and $m_{3}{ }^{\prime \prime}\left(m_{1}\right)(\mathrm{c}, \mathrm{f})$ for the biaxial EMNZ MM. Figure b details the data in Figure a at small values of $m_{3}{ }^{\prime}$, Figure e details the data in Figure d at small values of $m_{1}$.

solutions of equation (24) are designated by the different curve types in Figures 10-13. Only transmitted waves are considered $\left(m_{3}{ }^{\prime \prime}>0\right)$. The Figures $10-13$ data illustrate availability of two transmitted waves for each $m_{1}$ value, and all four (Fig. 10a-c and Fig. 13a) or only two (Fig. 10df, Figs. 11, 12 and 13b-e) solutions of equation (24) are used. For all the graphs in Figures 10-13, we use the values of the angles determining vectors $\boldsymbol{c}_{q}(q=1,2,3)$ in the chosen coordinate system: $\alpha_{1}=\gamma_{3}=\pi / 4, \alpha_{2}=\beta_{3}=3 \pi / 4$, $\alpha_{3}=\pi / 2, \beta_{1}=\beta_{2}=\gamma_{1}=\gamma_{2}=\pi / 3$.

Figure $10 \mathrm{a}-\mathrm{c}$, Figures 11 and 13a illustrate the effect of small dielectric birefringence (here the very small $\varepsilon^{\prime \prime}, \mu^{\prime \prime}$ values are taken for extraction of the transmitted waves), Figure $10 \mathrm{~d}-\mathrm{f}$, c and Figures 12, 13b-e characterize the mutual effect of birefringence and dichroism on dependences $m_{3}{ }^{\prime}\left(m_{1}\right), m_{3}{ }^{\prime \prime}\left(m_{1}\right), m_{3}{ }^{\prime}\left(m_{3}{ }^{\prime \prime}\right)$ for the biaxial MM. The graphs at the large scale of $m_{1}$ values in Figure 10 (that is, besides of Fig. 10e) correspond qualitatively to the case of the weakly anisotropic EMNZ MM (Fig. 6b and c). In this case, even very small birefringence parameters (of the order of $10^{-5}$, Fig. 10a and b) lead to the phase shifts that exceed in 5-6 orders and greater the corresponding values for the isotropic MM (Fig. 1). The growth of the dielectric and magnetic anisotropy parameters (Fig. 10d and e) leads to more stronger $m_{3}{ }^{\prime}\left(m_{1}\right)$ dependences and nonlinearity of these dependences at small $m_{1}$ values (Fig. 10e). It is significant that dependences $m_{3}{ }^{\prime \prime}\left(m_{1}\right)$ are appeared to be "steady" to MM small anisotropy (Fig. 10c, f, and 11d, the similar graphs take place also for the data in Fig. 12).

For the nonabsorbing SL MM (Fig. 11, the data in Fig. 11a at small $m_{3}{ }^{\prime}$ and $m_{1}$ values are specified by the graphs in Fig. $11 \mathrm{~b}$ and $\mathrm{c}$ ), the presence of small birefringence of the order of $10^{-5}$ leads to the qualitative changes of dependences $m_{3}{ }^{\prime}\left(m_{1}\right)$ in comparison with the isotropic SL case (Fig. 3). In particular, the forward wave appears additionally to the backward wave in MM, with that, phase incursions of these waves rise with growing $\left|m_{1}\right|$ values (Fig. 11b). At the same parameters and $\mu_{q}{ }^{\prime}=1$ (the case of NMSL), the function $m_{3}{ }^{\prime \prime}\left(m_{1}\right)$ graphs (Fig. 11d) do not change practically. In this case, dependences $m_{3}{ }^{\prime}\left(m_{1}\right)$ correspond to Fig. 11b (but without the features in the diapason $m_{1} \in(-1,1)$, the graphs are near-linear). The similar characteristic properties take place also at small deviations of the same order from the values $\varepsilon_{q}{ }^{\prime}=-1$ to the larger values.

In the case of SL MM (Fig. 12a), the presence of dichroism leads to $m_{3}{ }^{\prime}\left(m_{1}\right)$ dependences that are practically symmetric with respect to the axis $m_{1}=0$ and become stronger with the growth of quantities $\left|\varepsilon_{2}{ }^{\prime \prime}-\varepsilon_{1}{ }^{\prime \prime}\right|, \mid \varepsilon_{3}{ }^{\prime \prime}-$ $\varepsilon_{1}{ }^{\prime \prime} \mid$ (determined by parameter $p$ ). These dependences are near-linear at the values $\left|m_{1}\right|>5-10$. As for the uniaxial MM (Figs. 7 and 8), birefringence (characterized in this case by quantities $\left.\left|\varepsilon_{2}{ }^{\prime}-\varepsilon_{1}{ }^{\prime}\right|,\left|\varepsilon_{3}{ }^{\prime}-\varepsilon_{1}{ }^{\prime}\right|\right)$ determines the asymmetry of the function $m_{3}^{\prime}\left(m_{1}\right)$ graphs with respect to the parameter $m_{1}$ sign (Fig. 12b). Small dichroism and anisotropy (of the order of $10^{-4}-10^{-3}$ ) in the considered MM lead to the phase shift values corresponding to rather large absorption in the isotropic MM (Fig. 1). At the small scale of $\left|m_{1}\right|$ values, the graphs in Figure 12a and b are practically coincident and correspond to the data in Figure 12c. The similar features take place also in the presence of small magnetic anisotropy of the same order and at the $\varepsilon_{q}, \mu_{q}$ values near to $-1,1$ correspondingly (the NMSL case).

The Figures 10 and 12 data are illustrated by Figure 13 in axes $\left(m_{3}{ }^{\prime}, m_{3}{ }^{\prime \prime}\right)$. In the case of EMNZ MM, the presence of only small birefringence leads to near-linear dependences $m_{3}^{\prime}\left(m_{3}{ }^{\prime \prime}\right)$ for both the large (Fig. 13a) and small scale of $\left|m_{1}\right|$ values. With the growth of the dielectric and magnetic anisotropy parameters, dependences $m_{3}{ }^{\prime}\left(m_{3}{ }^{\prime \prime}\right)$ became significantly stronger (Fig. 13b) and nonlinear at small values of $m_{1}$ (Fig. 13c). In the case of SL MM (Fig. 13d and e), the mutual effect of birefringence and dichroism also leads to the nonlinear graphs for both small and large absorption corresponding to the small and large values of parameter $\left|m_{1}\right|$. In the given case (as for the uniaxial MM), the different scale of parameter $m_{3}{ }^{\prime}$ changes takes place (the difference is approximately in a three order of magnitude) for EMNZ (Fig. 13a and b) and SL (Fig. 13d and e) MM at the comparable variations of the anisotropy parameters.

The features of the dispersion dependences of uniaxial (Figs. 6-9) and biaxial (Figs. 10-13) MM are characteristic and take place also at other MM material parameters, directions of the optical axis for the uniaxial MM and orientations of vectors $\boldsymbol{c}_{q}$ for the biaxial MM.

\section{Conclusion}

The executed analysis points to the crucial role of effective dielectric and magnetic anisotropy (in comparison with absorption) in forming the undesirable phase incursion of electromagnetic waves in the MM applications. The presence of even a very small anisotropy leads generally to near-linear (piecewise-linear) dependences of the phase shift on the transversal wave number (the phase incursion increases 


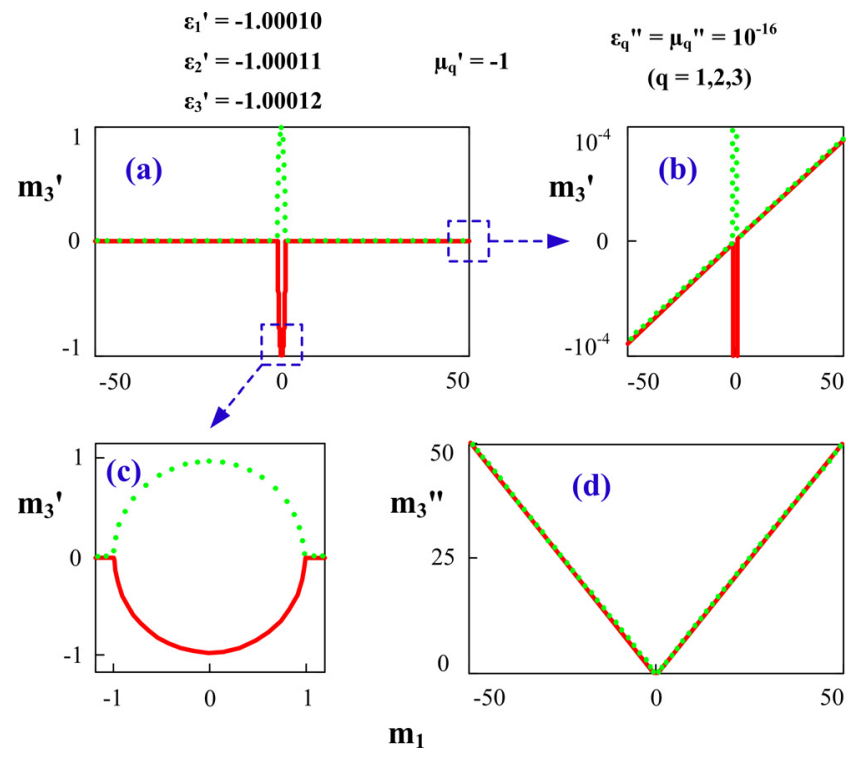

Fig. 11. The effect of small birefringence on dependences $m_{3}{ }^{\prime}\left(m_{1}\right)$ (a-c) and $m_{3}{ }^{\prime \prime}\left(m_{1}\right)$ (d) for the biaxial SL MM. Figures $\mathrm{b}$ and $\mathrm{c}$ detail the data in Figure a at small values of $m_{3}{ }^{\prime}$ and $m_{1}$, correspondingly.

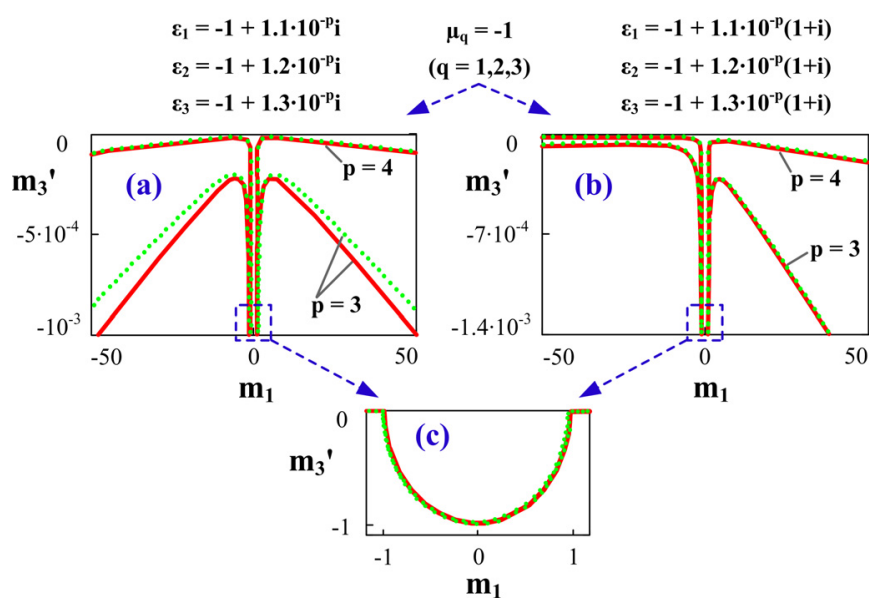

Fig. 12. The effect of small dichroism (a), the mutual effect of birefringence and dichroism (b) on dependences $m_{3}{ }^{\prime}\left(m_{1}\right)$ for the biaxial SL MM. Figure c corresponds to the cases $(\mathrm{a}, \mathrm{b})$ at small values of $m_{1}$.

quickly with the growth of values $\left.\left|m_{1}\right|\right)$. The characteristics of wave attenuation for the absorbing isotropic and weakly anisotropic MM are comparable (the function $m_{3}{ }^{\prime \prime}\left(m_{1}\right)$ graphs at the large scale of $m_{1}$ values are weakly discriminated for all the considered cases excepting some peculiar cases, Fig. 6b). However, very small (and so hardly controllable for real MM) anisotropy (of the order of $|\Delta \varepsilon|,|\Delta \mu| \approx 10^{-5}-10^{-3}$ ) leads to the same phase shifts as ones caused by rather large absorption in the isotropic MM (of the order of $\varepsilon^{\prime \prime}, \mu^{\prime \prime} \approx 0.1$ and larger) in the range of "working values" of $m_{1}$. That causes a quick violation of "ideal" conditions for the MM applications: the image deterioration and withdrawal from the "superlensing" regime for SL (NMSL), unwanted misphasing of the waves for EMNZ (ENZ) MM.
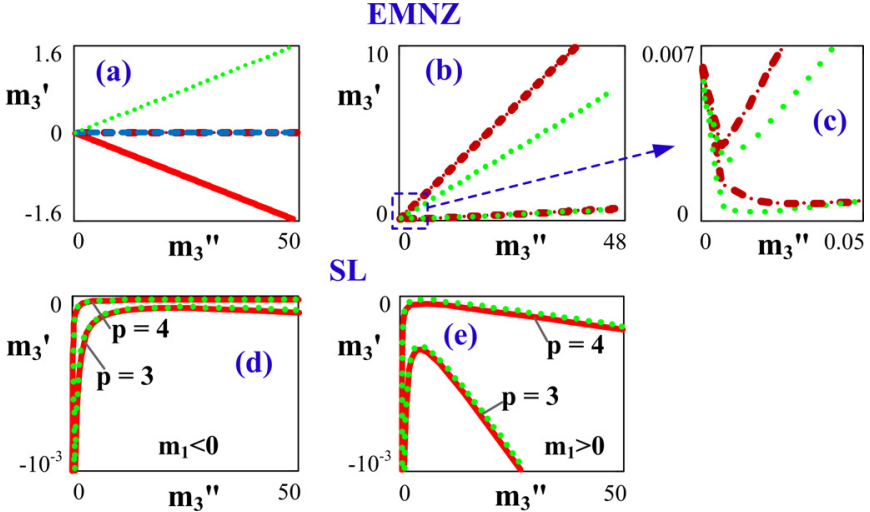

SL

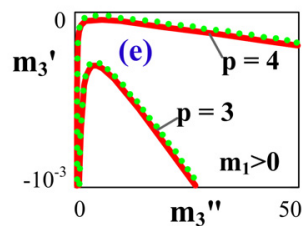

Fig. 13. Dependences $m_{3}{ }^{\prime}\left(m_{3}{ }^{\prime \prime}\right)$ for the data given in Figures 10, 12. The data correspondence is: a - Figures $10 \mathrm{a}-\mathrm{c} ; \mathrm{b}, \mathrm{c}-$ Figures 10d-f; d, e - Figures $12 \mathrm{~b}, \mathrm{c}$ (at $m_{1}<0$ and $m_{1}>0$ ). The same line types are used for the corresponding data in Figures 10, 12 and 13. Figure $\mathrm{c}$ details the data in Figure $\mathrm{b}$ at small values of $m_{3}{ }^{\prime \prime}$.

So, one of the conclusions of paper [20] (where uniaxial $\mathrm{MM}$ and the realization of SL were considered) about the crucial influence of small anisotropy on the SL operability can be significantly generalized. Small electromagnetic anisotropy of a general form (dielectric, magnetic, uniaxial, biaxial) is the universal "non-ideal" factor determining (to a much greater extent than small losses) the operability of SL (NMSL), EMNZ (ENZ) MM and, apparently, also the other MM applications where the wave misphasing in the effective medium is undesirable. The presence of effective anisotropy (either required for applications or unwanted) is the feature of many realized MM. Therefore, the careful account of both losses and even a very small permittivity (permeability) anisotropy for various MM applications is necessary. Besides the comparative analysis of dispersion equations for MM with various anisotropic properties, a number of important particular cases (that can be of interest for optimization of the MM applications) has been investigated.

Obviously, the obtained results accounting mainly the dispersion equations can be considered only as initial estimations of the operability of "non-ideal" MM applications. For a more detailed analysis, e.g., of SL MM, one can determine the fields in the image region or transfer functions for the concrete SL devices $[3,17,19,26,35]$ and consider the change of the images with increasing absorption and anisotropy parameters. However, the ascertained features of the wave misphasing and damping in MM are rather general ("from first principles"). So, they will appear also in cases of a more detailed consideration of the concrete devices using MM. The results can be useful also in the investigations of interaction of electromagnetic fields of a more complicated structure (wave beams, waveguide modes, et al.) with MM for the cases when these fields are represented by monochromatic waves superpositions.

Moreover, one can note the limitations of the traditional approaches using the second-order curves (or surfaces) for analytical modeling of the dispersion curves (surfaces) of absorbing MM. The obtained analytical and numerical results illustrate clearly that even a very small electromag- 
netic absorption and anisotropy lead to the cardinal changes of complex solutions of the second-order dispersion equations. Even for the simplest cases of the absorbing isotropic and uniaxial media (including MM), dependences $m_{3}{ }^{\prime}\left(m_{1}\right)$ and $m_{3}{ }^{\prime \prime}\left(m_{1}\right)$ are characterized by the fourth-order curves, and these curves (surfaces) have even higher than four orders in the case of absorbing biaxial media. Rise of the order of the dispersion dependences complicates appreciably MM investigations but leads to more realistic and useful models of MM and devices on the basis of MM.

\subsection{Implications and influences}

On the basis of the detailed analysis "from first principles", it is established that even a very small (hundredths of a percent and less) effective electromagnetic anisotropy is the universal "non-ideal" factor determining (to a much greater extent than small losses) the operability of the MM applications ("zero" media, "superlens"). So, a careful account of both the material absorption and even small permittivity (permeability) anisotropy of the general form for various MM applications is necessary. Limitations of the traditional approaches using the second-order dispersion curves (or surfaces) for analytic modeling of the absorbing electromagnetic MM with local material parameters are shown. It is required to use the dispersion equations of the fourth and higher orders as more realistic and useful models of the considered MM.

\section{Appendix}

The coefficients of equation (24) can be represented in the form:

$$
\begin{aligned}
& A=\left(Q\left(\gamma_{1}, \gamma_{2}\right)+\varepsilon_{3} P\left(\gamma_{1}, \gamma_{2}\right)\right)\left(M\left(\gamma_{1}, \gamma_{2}\right)\right. \\
&+\left.\mu_{3} P\left(\gamma_{1}, \gamma_{2}\right)\right) \\
& B= 2 m_{1}\left[\left(F\left(t_{1}, t_{2}\right)-2 \varepsilon_{3} \mu_{3} F(1,1)\right) P\left(\gamma_{1}, \gamma_{2}\right)\right. \\
&-G\left(\gamma_{1}, \gamma_{2}\right) F(1,1)+F\left(\mu_{1}, \mu_{2}\right) Q\left(\gamma_{1}, \gamma_{2}\right) \\
&\left.+M\left(\gamma_{1}, \gamma_{2}\right) F\left(\varepsilon_{1}, \varepsilon_{2}\right)\right] \\
& C=\left(m_{1}^{2} M\left(\alpha_{1}, \alpha_{2}\right)-\varepsilon_{3} \mu_{1} \mu_{2}\right) Q\left(\gamma_{1}, \gamma_{2}\right)+\left(m_{1}^{2} Q\left(\alpha_{1}, \alpha_{2}\right)\right. \\
&\left.-\mu_{3} \varepsilon_{1} \varepsilon_{2}\right) M\left(\gamma_{1}, \gamma_{2}\right)+\left(m_{1}^{2} G\left(\alpha_{1}, \alpha_{2}\right)\right. \\
&\left.\quad-\varepsilon_{3} \mu_{3} t_{3}\right) P\left(\gamma_{1}, \gamma_{2}\right)+m_{1}^{2}\left[4 F\left(\mu_{1}, \mu_{2}\right) F\left(\varepsilon_{1}, \varepsilon_{2}\right)\right. \\
&-4 F\left(t_{1}, t_{2}\right) F(1,1)+G\left(\gamma_{1}, \gamma_{2}\right) P\left(\alpha_{1}, \alpha_{2}\right) \\
&\left.+2 \varepsilon_{3} \mu_{3}\left(P\left(\alpha_{1}, \alpha_{2}\right) P\left(\gamma_{1}, \gamma_{2}\right)+2 F^{2}(1,1)\right)\right] \\
& \quad \\
& D=2 m_{1}^{3} P\left(\alpha_{1}, \alpha_{2}\right)\left(F\left(t_{1}, t_{2}\right)-2 \varepsilon_{3} \mu_{3} F(1,1)\right) \\
& \quad+2 m_{1}\left[\left(m_{1}^{2} M\left(\alpha_{1}, \alpha_{2}\right)-\varepsilon_{3} \mu_{1} \mu_{2}\right) F\left(\varepsilon_{1}, \varepsilon_{2}\right)\right. \\
&+\left(m_{1}^{2} Q\left(\alpha_{1}, \alpha_{2}\right)-\mu_{3} \varepsilon_{1} \varepsilon_{2}\right) F\left(\mu_{1}, \mu_{2}\right) \\
&\left.-\left(m_{1}^{2} G\left(\alpha_{1}, \alpha_{2}\right)-\varepsilon_{3} \mu_{3} t_{3}\right) F(1,1)\right]
\end{aligned}
$$

$$
\begin{aligned}
& E=m_{1}^{4} \varepsilon_{3} \mu_{3} P^{2}\left(\alpha_{1}, \alpha_{2}\right)+m_{1}^{2} P\left(\alpha_{1}, \alpha_{2}\right)\left(m_{1}^{2} G\left(\alpha_{1}, \alpha_{2}\right)\right. \\
& \left.-\varepsilon_{3} \mu_{3} t_{3}\right)+\left(m_{1}^{2} M\left(\alpha_{1}, \alpha_{2}\right)\right. \\
& \left.-\varepsilon_{3} \mu_{1} \mu_{2}\right)\left(m_{1}^{2} Q\left(\alpha_{1}, \alpha_{2}\right)-\mu_{3} \varepsilon_{1} \varepsilon_{2}\right),
\end{aligned}
$$

where the denotations are used: $t_{1}=\varepsilon_{3} \mu_{1}+\varepsilon_{1} \mu_{3}, t_{2}=$ $\varepsilon_{3} \mu_{2}+\varepsilon_{2} \mu_{3}, \quad t_{3}=\varepsilon_{1} \mu_{2}+\varepsilon_{2} \mu_{1}, \quad Q\left(\varphi_{1}, \varphi_{2}\right)=\varepsilon_{1} \cos ^{2}\left(\varphi_{1}\right)+$ $\varepsilon_{2} \cos ^{2}\left(\varphi_{2}\right), \quad M\left(\varphi_{1}, \quad \varphi_{2}\right)=\mu_{1} \cos ^{2}\left(\varphi_{1}\right)+\mu_{2} \cos ^{2}\left(\varphi_{2}\right)$, $P\left(\varphi_{1}, \varphi_{2}\right)=1-\cos ^{2}\left(\varphi_{1}\right)-\cos ^{2}\left(\varphi_{2}\right), \quad F(x, y)=x \cos \left(\alpha_{1}\right) \cos$ $\left(\gamma_{1}\right)+y \cos \left(\alpha_{2}\right) \cos \left(\gamma_{2}\right), G\left(\varphi_{1}, \varphi_{2}\right)=t_{1} \cos ^{2}\left(\varphi_{1}\right)+t_{2} \cos ^{2}\left(\varphi_{2}\right)$. According to expressions (A1)-(A5), coefficient $A$ does not depend on parameter $m_{1}$, and coefficients $B, C, D, E$ are incomplete polynomials of the orders $1,2,3$, 4 with respect to $m_{1}$, correspondingly.

\section{References}

1. V.G. Veselago, Sov. Phys. Usp. 10, 509 (1968) (Russian text: Usp. Fiz. Nauk 92, 517 (1967))

2. D.R. Smith et al., Phys. Rev. Lett. 84, 4184 (2000)

3. J.B. Pendry, Phys. Rev. Lett. 85, 3966 (2000)

4. A.M. Urbas, Z. Jacob, L.D. Negro et al., J. Opt. 18, 093005 (2016)

5. M. Decker, I. Staude, J. Opt. 18, 103001 (2016)

6. Z. Wang et al., Nanotechnology 27, 412001 (2016)

7. S. Jahani, Z. Jacob, Nat. Nanotechnol. 11, 23 (2016)

8. T. Cui, S. Liu, L. Zhang, J. Mater. Chem. C 5, 3644 (2017)

9. I. Liberal, N. Engheta, Nat. Photonics 11, 149 (2017)

10. S.A. Tretyakov, J. Opt. 19, 013002 (2017)

11. A.D. Boardman, P. Egan, M. McCall, EPJ Appl. Metamater. 2, 11 (2015)

12. A. Poddubny, I. Iorsh, P. Belov, Y. Kivshar, Nat. Photonics 7, 948 (2013)

13. V.M. Shalaev, Nat. Photonics 1, 41 (2007)

14. C.M. Soukoulis, M. Wegener, Nat. Photonics, 5, 523 (2011)

15. T. Xu et al., Nature 497, 470 (2013)

16. P.F. Loschialpo et al., Phys. Rev. E 70, 036605 (2004)

17. W. Cai, D.A. Genov, V.M. Shalaev, Phys. Rev. B 72, 193101 (2005)

18. R.S. Hegde et al., IEEE Trans. microw. theory tech. 59, 2612 (2011)

19. A.P. Vinogradov, A.V. Dorofeenko, Opt. Commun. 256, 333 (2005)

20. E. Starodubtsev, Proc. SPIE 7353, 73530A (2009)

21. R.W. Ziolkowski, Phys. Rev. E 70, 046608 (2004)

22. A. Alù, M.G. Silveirinha, A. Salandrino, N. Engheta, Phys. Rev. B 75, 155410 (2007)

23. R. Maas, J. Parsons, N. Engheta, A. Polman, Nat. Photonics 7, $907(2013)$

24. Y. Li et al., Nat. Photonics 9, 738 (2015)

25. Z. Liu, L. Hu, Z. Lin, Phys. Lett. A 308, 294 (2003)

26. T. Dumelow, J.A. Pereira da Costa, V.N. Freire, Phys. Rev. B 72, 235115 (2005)

27. S. Qiao, G. Zheng, H. Zhang, L. Ran, PIER 81, 267 (2008)

28. L. Sun, S. Feng, X. Yang, Appl. Phys. Lett. 101, 241101 (2012)

29. K.E. Ballantine, J.F. Donegan, P.R. Eastham, Phys. Rev. A 90, $013803(2014)$

30. E.G. Starodubtsev, Metamaterials 4, 32 (2010) 
31. F.I. Fedorov, Optics of Anisotropic Media, 2nd edn. (Editorial URSS, Moscow, 2004 (in Russian))

32. E. Starodubtsev, Proc. SPIE 6581, 65810Z (2007)

33. N.S. Petrov, F.I. Fedorov, Opt. i Spektrosk. 15, 792 (1963) (in Russian)
34. F.I. Fedorov, Theory of gyrotropy (Nauka i Teknika, Minsk, 1976 (in Russian))

35. M. Lapine, L. Jelinek, M.J. Freire, R. Marques, Phys. Rev. B 82, $165124(2010)$

Cite this article as: Evgenii Starodubtsev, Effect of small anisotropy and absorption on metamaterial applications: "non-ideal" features of propagation and tunneling of electromagnetic waves, EPJ Appl. Metamat. 2018, 5, 1 